\title{
The OTT-MAL fusion oncogene activates RBPJ-mediated transcription and induces acute megakaryoblastic leukemia in a knockin mouse model
}

\author{
Thomas Mercher, ${ }^{1,2,3}$ Glen D. Raffel,1 Sandra A. Moore,1 Melanie G. Cornejo,1 \\ Dominique Baudry-Bluteau, ${ }^{2}$ Nicolas Cagnard, ${ }^{3}$ Jonathan L. Jesneck, ${ }^{4}$ Yana Pikman, ${ }^{1}$ \\ Dana Cullen, ${ }^{1}$ Ifor R. Williams, ${ }^{5}$ Koichi Akashi, ${ }^{4}$ Hirokazu Shigematsu, ${ }^{4}$ \\ Jean-Pierre Bourquin, ${ }^{6}$ Marco Giovannini, ${ }^{7}$ William Vainchenker, ${ }^{8}$ Ross L. Levine, ${ }^{1}$ \\ Benjamin H. Lee, ${ }^{1}$ Olivier A. Bernard,2,3 and D. Gary Gilliland ${ }^{1,9}$
}

\begin{abstract}
1Division of Hematology, Department of Medicine, Brigham and Women's Hospital, Harvard Medical School, Boston, Massachusetts, USA. ${ }_{2}^{2}$ INSERM EMI0210, Paris, France. 3Université René Descartes, Hôpital Necker, Paris, France. ${ }^{4}$ Dana-Farber Cancer Institute, Boston, Massachusetts, USA. ${ }^{5}$ Department of Pathology, Emory University School of Medicine, Atlanta, Georgia, USA. ${ }^{6}$ Department of Oncology, Children's Hospital, University of Zurich, Zurich, Switzerland. 7INSERM U674, Fondation Jean Dausset - CEPH, Paris, France.

8INSERM U790, Institut Gustave Roussy, Université Paris-Sud 11, Villejuif, France. ${ }^{9}$ Howard Hughes Medical Institute, Cambridge, Massachusetts, USA.
\end{abstract}

\begin{abstract}
Acute megakaryoblastic leukemia (AMKL) is a form of acute myeloid leukemia (AML) associated with a poor prognosis. The genetics and pathophysiology of AMKL are not well understood. We generated a knockin mouse model of the one twenty-two-megakaryocytic acute leukemia (OTT-MAL) fusion oncogene that results from the $t(1 ; 22)(\mathrm{p} 13 ; \mathrm{q} 13)$ translocation specifically associated with a subtype of pediatric AMKL. We report here that OTT-MAL expression deregulated transcriptional activity of the canonical Notch signaling pathway transcription factor recombination signal binding protein for immunoglobulin $\kappa \mathrm{J}$ region (RBPJ) and caused abnormal fetal megakaryopoiesis. Furthermore, cooperation between OTT-MAL and an activating mutation of the thrombopoietin receptor myeloproliferative leukemia virus oncogene (MPL) efficiently induced a short-latency AMKL that recapitulated all the features of human AMKL, including megakaryoblast hyperproliferation and maturation block, thrombocytopenia, organomegaly, and extensive fibrosis. Our results establish that concomitant activation of RBPJ (Notch signaling) and MPL (cytokine signaling) transforms cells of the megakaryocytic lineage and suggest that specific targeting of these pathways could be of therapeutic value for human AMKL.
\end{abstract}

\section{Introduction}

Acute megakaryoblastic leukemia (AMKL) is a heterogeneous subtype of acute myeloid leukemia (AML) and is more frequent in children than in adults (1-3). The molecular basis of AMKL is poorly understood in adults, whereas 2 major molecular subtypes are recognized in pediatric AMKL. The first group is represented by Down syndrome (DS) patients with both transient myeloproliferative disease (transient MPD) and AMKL who present with acquired GATA-binding protein 1 (GATA1) mutations, resulting in an N-terminal truncated GATA1 short (GATA1s) protein (4). The second group occurs in infants and is associated with the $\mathrm{t}(1 ; 22)(\mathrm{p} 13 ; \mathrm{q} 13)$ chromosomal translocation, resulting in expression of the one twenty-two megakaryocytic acute leukemia (OTT-MAL) (also known as RBM15-MKL1) fusion protein (5-7).

Conflict of interest: The authors have declared that no conflict of interest exists. Nonstandard abbreviations used: AchE, acetylcholinesterase; AMKL, acute megakaryoblastic leukemia; CMP, common myeloid progenitor; dn, dominant negative; DS, Down syndrome; GATA1, GATA-binding protein 1; GEMM, granulocyte-macrophage-erythroid-Mk; GSEA, Gene Set Enrichment Analysis; LSK, Lineage-Sca1+c-Kit ${ }^{+}$; MAML1, Mastermind-like 1; MEP, Mk-erythrocyte progenitor; Mk, megakaryocyte; MkP, Mk progenitor; MPD, myeloproliferative disease; MPL, myeloproliferative leukemia virus oncogene; OM, Ott ${ }^{O t t-M A L / W T}$; OTT-MAL, one twenty-two megakaryocytic acute leukemia; RBPJ, recombination signal binding protein for immunoglobulin $\kappa \mathrm{J}$ region; RRM, RNA recognition motif; TAD, transactivation domain; TNR, transgenic Notch reporter; TPO, thrombopoietin.

Citation for this article: J. Clin. Invest. 119:852-864 (2009). doi:10.1172/JCI35901.
MAL is a cofactor of the serum response factor (SRF) endowed with strong transcriptional activation properties (8), and its localization and function are deregulated by fusion with OTT (9). OTT is related to the SHARP transcription factor that has been shown to interact with recombination signal binding protein for immunoglobulin $\mathrm{KJ}$ region (RBPJ), the transcription factor involved in response to the canonical Notch pathway, and repress transcription of its target genes in absence of Notch signaling (10-13).

The thrombopoietin (TPO) receptor myeloproliferative leukemia virus oncogene (MPL) and its ligand, TPO, are essential for the proliferation of megakaryocyte (Mk) progenitors $(\mathrm{MkPs})$ and their differentiation into mature platelet-producing Mks (14). Ligand stimulation of MPL results in activation of the Janus kinases JAK2 and TYK2, followed by docking and phosphorylation of a spectrum of signaling proteins (15-17). The relevance of this pathway to pathogenesis of AMKL is highlighted by demonstration of activating point mutations in JAK2 or JAK3 (18-20) and in MPL (21). In addition, secondary AMKL may also occur with leukemic transformation of chronic myeloproliferative syndromes, including polycythemia vera, essential thrombocytosis, and myeloid metaplasia, that are frequently associated with activating mutations in JAK2 or MPL $(22,23)$. These observations indicate that activating mutations in the $\mathrm{TPO} / \mathrm{MPL} / \mathrm{JAK}$ signaling pathway play an important role in $\mathrm{Mk}$ malignancies in humans. 
A
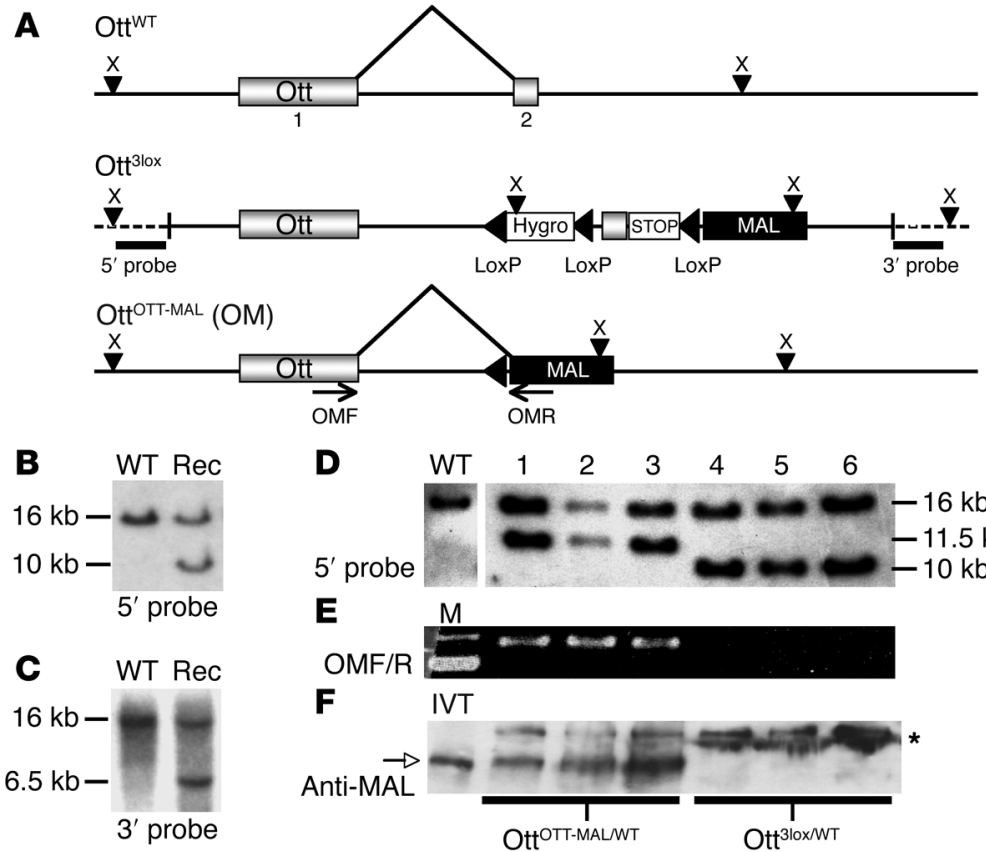

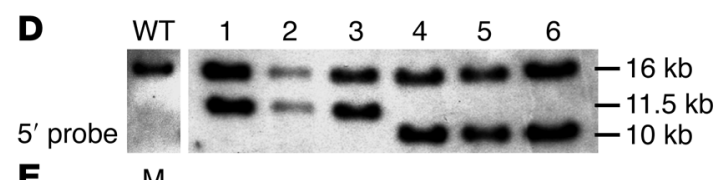

E $M$

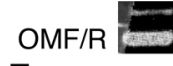

$\mathbf{F}$

Anti-MAL

IVT $\cdots \infty$

\section{Figure 1}

Ott-MAL knockin approach. (A) Mouse Ott locus (showing splicing between exon 1 and 2), OTT-MAL targeting construct/Ott ${ }^{3 l o x}$ allele, and OM allele are represented. OMF and OMR show locations of primers used for detection of the fusion transcript by RT-PCR. X, Xmnl site. (B and $\mathbf{C}$ ) Southern blot analysis of ES clone DNA using $\mathrm{Xmnl}$ digestion show correct homologous recombination on the $5^{\prime}$ and $3^{\prime}$ sides of the targeting construct, respectively. Rec, recombined. (D-F) Southern blot, RT-PCR, and Western blot analysis, respectively, on ES clones obtained after adeno-Cre-mediated excision of the Hygro-STOP cassette. Arrow indicates the OTT-MAL (OM) fusion protein. Asterisk indicates nonspecific band; IVT, in vitro transcription control.
To gain insight into the requirements for AMKL leukemogenesis, we generated a conditional knockin allele of Ott-MAL in mice. We observe that OTT-MAL expression from its endogenous promoter results in aberrant megakaryopoiesis associated with enhanced self renewal and activation of RBPJ-mediated transcription. In addition, analysis of gene expression data from infants with AMKL supports a role for deregulation of Notch signaling in transformation of the megakaryocytic lineage. We further observe that expression of OTT-MAL with concomitant Notch signaling deregulation cooperates with an activating allele of MPL to induce a highly penetrant AMKL that recapitulates the human phenotype.

\section{Results}

Conditional expression of Ott-MAL in a knockin mouse model. We engineered a conditional Ott-MAL knockin mouse model that allowed for expression of the fusion protein from the endogenous murine Ott promoter after Cre recombinase-mediated excision of a STOP cassette flanked by loxP recombination sites (floxed-STOP; Figure 1A). Southern blot analysis of XmnI-digested ES cell DNA identified clones with homologous recombination (Figure 1, B and C). Functional integrity of the targeted construct was confirmed by transducing $O t t^{3 l o x} / W T$ ES clones with adenovirus encoding the Cre recombinase, followed by selection of $\mathrm{Ott}{ }^{\mathrm{Ot}-M A L}$ excised clones based on Southern blot analysis (Figure 1D). Conditional expression of the fusion mRNA and protein was confirmed by RT-PCR and Western blot analysis, respectively (Figure 1, E and F). Animals with germline transmission of the $\mathrm{Ott}^{3 l o x}$ allele were crossed to an EIIA-Cre transgenic mouse line, resulting in in vivo excision of the floxed-STOP cassette, with germline transmission of the Ott $\mathrm{Ott}^{\mathrm{O}-M A L}$ allele. Ott ${ }^{O t t-M A L / W T}$ animals (termed OM) were observed at predicted Mendelian ratios and were backcrossed into the C57BL/6 background for further analysis.

OTT-MAL expression results in abnormal fetal and adult hematopoiesis but induces AMKL with low penetrance. As the OTT-MAL fusion is restricted to infant leukemias and is likely to occur in utero, we first analyzed fetal liver hematopoiesis using flow cytometry. Plating of purified Lineage-Sca $1^{+} \mathrm{c}-\mathrm{Kit}^{+}$(LSK) cells from E12.5 fetal livers revealed a significant increase in colony-forming efficiency due to expansion of multipotent myeloid progenitors (CFU-granulocyte-macrophage-erythroid-Mk [CFU-GEMM]) in OM versus WT cells (Figure 2A), although no differences were observed in the absolute number of hematopoietic progenitors or CD41 $1^{+}$cells between OM and WT fetal liver at day E12.5 (Supplemental Figure 1; supplemental material available online with this article; doi:10.1172/JCI35901DS1; and data not shown). Furthermore, replating of LSK cell-derived primary colonies from OM embryos showed an increase in both Mk-containing (CFU-GEMM) and pure Mk (CFU-Mk) secondary colonies (Figure $2 \mathrm{~B}$ ), whereas no CFU-Mk were obtained with plating of WT cells. Identification of Mk-containing colonies was confirmed by staining cytospins of single colonies for acetylcholinesterase (AchE) activity (data not shown). These data indicate that OTT-MAL expression results in aberrant differentiation of HSC toward the megakaryocytic lineage in vitro.

We next investigated the effect of OTT-MAL expression on adult hematopoiesis. As OM animals did not show hematological abnormalities during the first 6 months of life (data not shown), we studied an older cohort of $15 \mathrm{OM}, 10 \mathrm{Ott}^{3 l o x} / \mathrm{WT}$, and 10 WT littermate animals aged 18-24 months. Since extramedullary hematopoiesis leading to splenomegaly is a hallmark of AMKL in humans, we first evaluated the number of progenitors in splenocytes and observed an increase in OM animals (Figure $2 \mathrm{C}$ ). In addition, these cells also showed enhanced replating activity in the majority of OM animals (8/15), whereas no replating activity was observed in cells derived from WT littermate $(0 / 10)$ or Ott $^{3 l o x}$ animals $(0 / 10)$ (Figure $\left.2 \mathrm{C}\right)$. Colonies derived from OM animals at the fifth round of replating showed $\mathrm{AchE}^{+}$ cells, indicating their megakaryocytic nature (data not shown). In addition, histopathologic analysis revealed that animals with increased replating activity of splenocytes had abnormalities in the hematopoietic system including extramedullary 
A

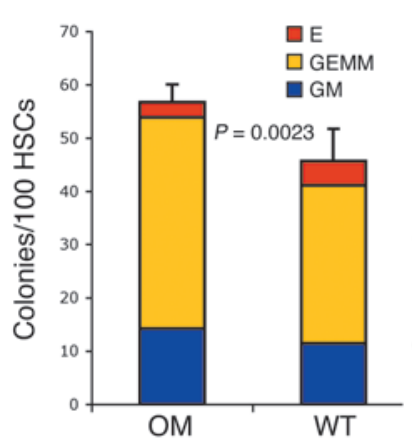

B

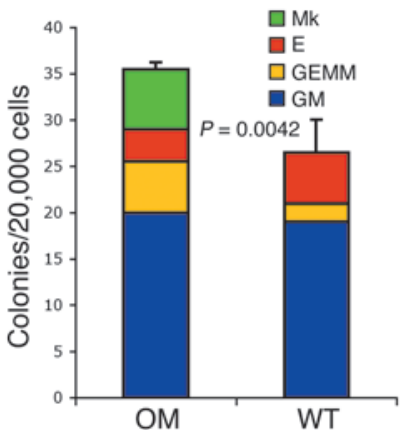

C

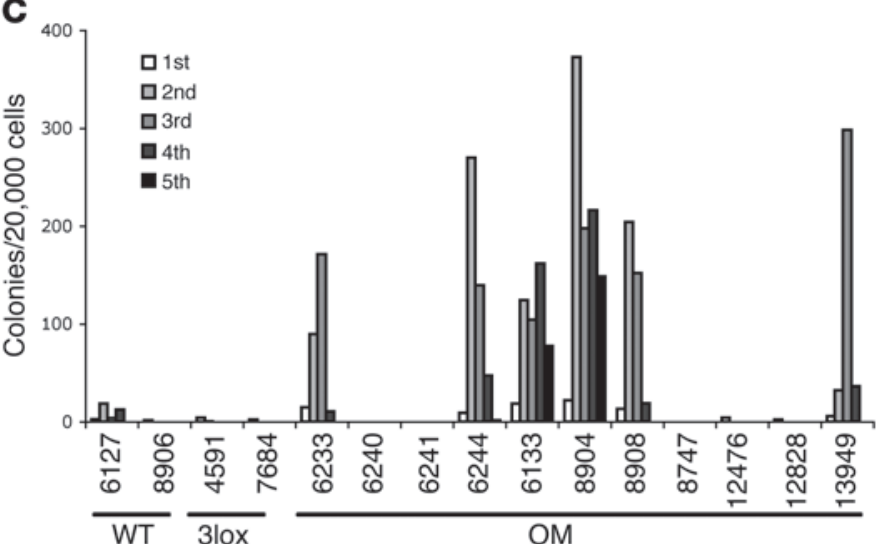

D

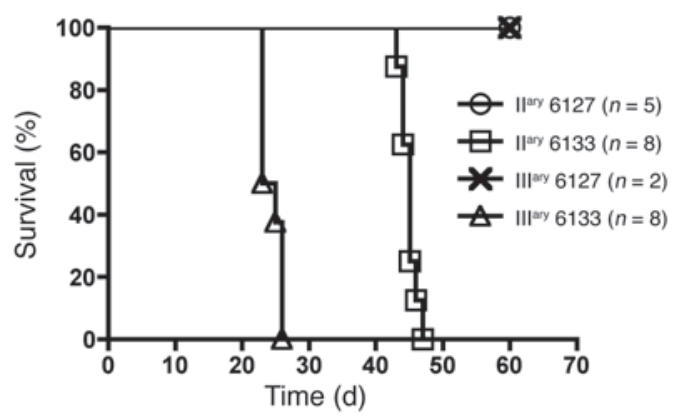

$\mathbf{F}$

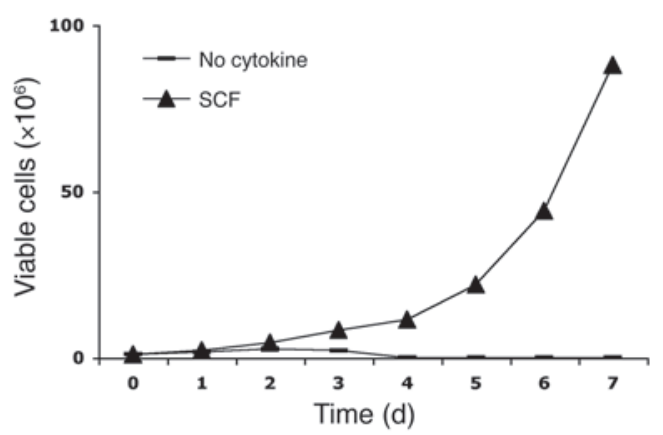

E

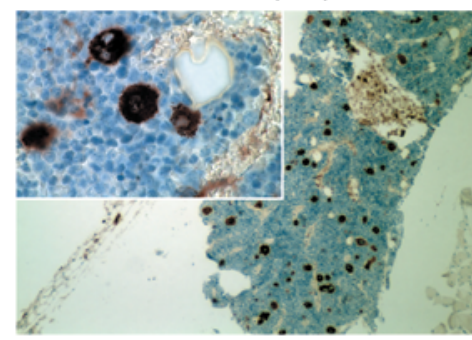

II ary $6133(\mathrm{OM})$

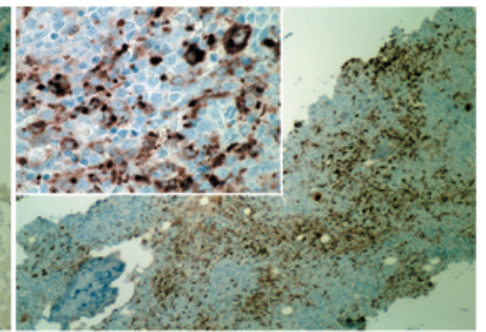

G

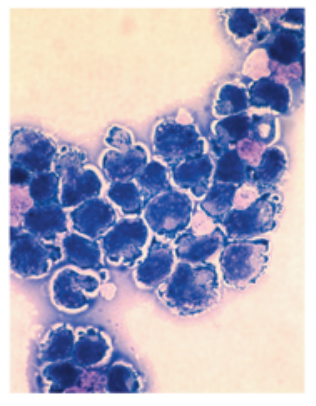

H
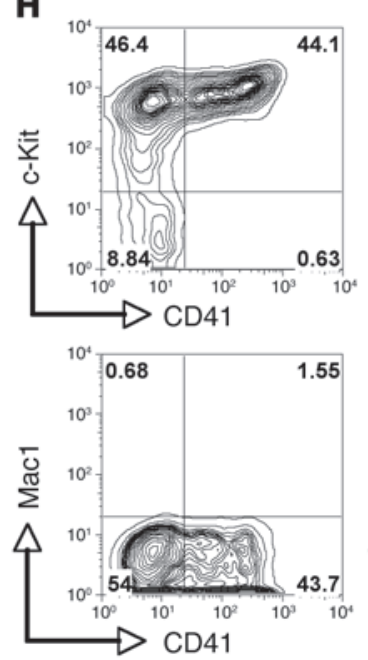
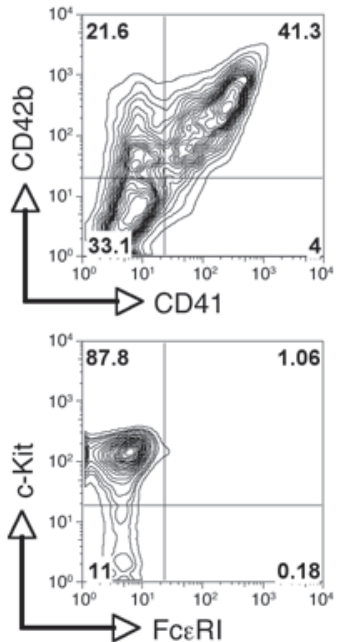

\section{Figure 2}

OTT-MAL induces abnormal fetal and adult hematopoiesis and AMKL with low penetrance. (A) Plating efficiency of LSK cells. Mean \pm SD of the number and type of colony counted after 7 days in triplicate is shown. (B) Colonies from A were replated into fresh methylcellulose plates and were scored 7 days later. Mean \pm SD of 3 independent experiments is shown. Mk, CFU-Mk; E, blast-forming unit-erythroid; GM, CFU-granulocyte-macrophage. (C) Serial replating assays of splenocytes from 18- to 24-month-old OM, Ott ${ }^{3 l o x} /$ WT , and WT littermate animals. Mean value of the number of colonies is indicated. (D) Kaplan-Meier survival curves of secondary (IIary) and tertiary (IIlary) recipients injected with $1 \times 10^{6}$ splenocytes from 6127 (WT) and 6133 (OM). (E) Immunohistochemistry on BM sections from secondary recipients using vWF antibody. Cells expressing vWF show a dark brown coloration. Original magnification, $\times 100 ; \times 1000$ (insets). (F) Proliferation of 6133 cells in absence or presence of SCF $(10 \mathrm{ng} / \mathrm{ml})$. Of note, proliferation of 6133 cells was not dependent on TPO. (G) Wright-Giemsa staining of the 6133 cell line. Original magnification, $\times 1000$. (H) Flow cytometrical immunophenotype of 6133 cells. Percentages of live cells are indicated. 
A

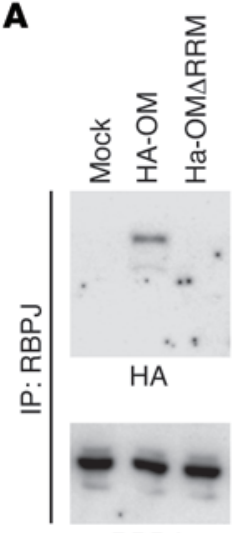

RBPJ

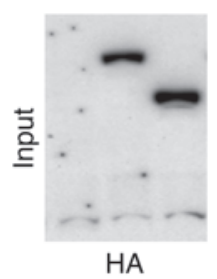

B
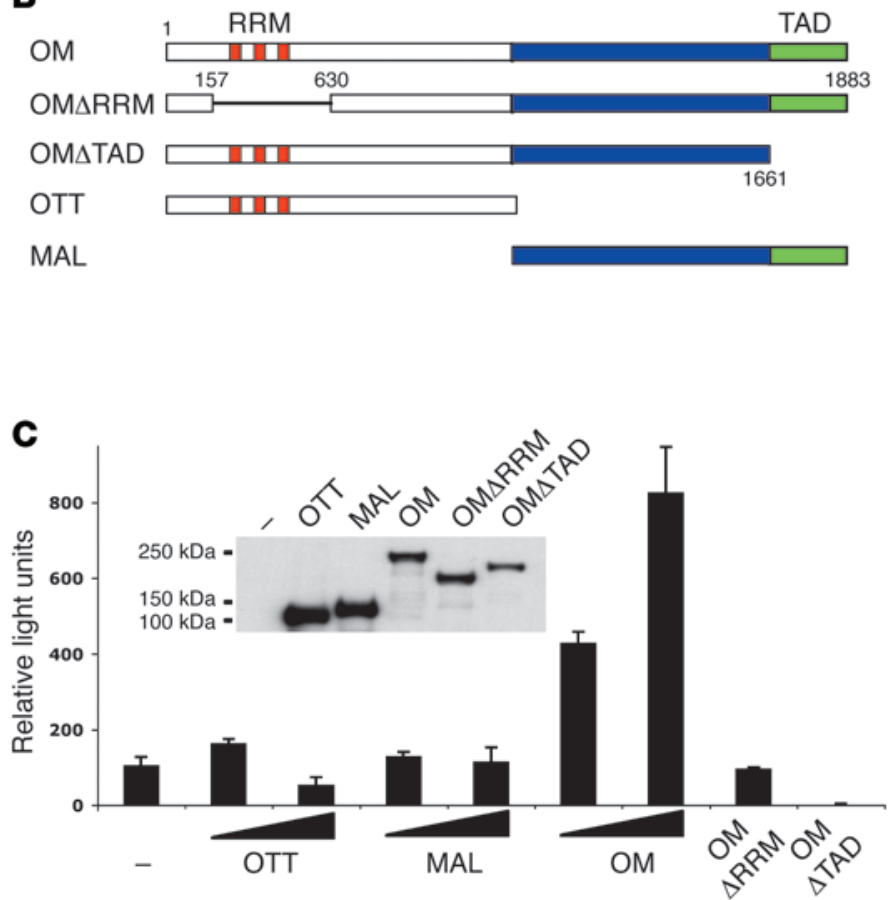

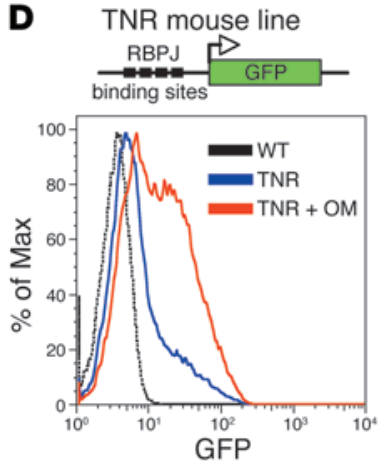

E

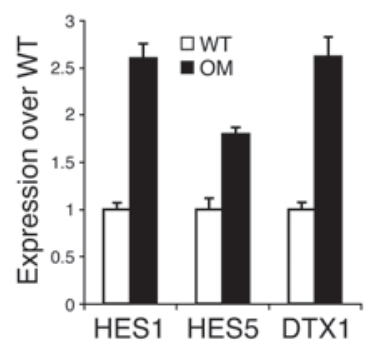

$\mathbf{F}$

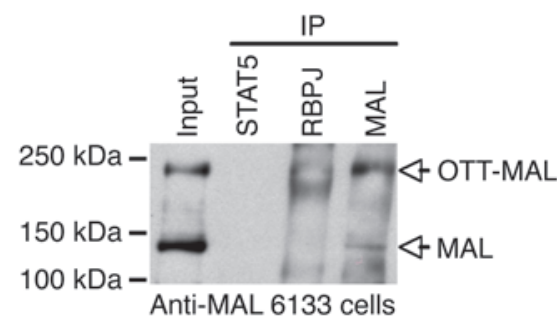

G

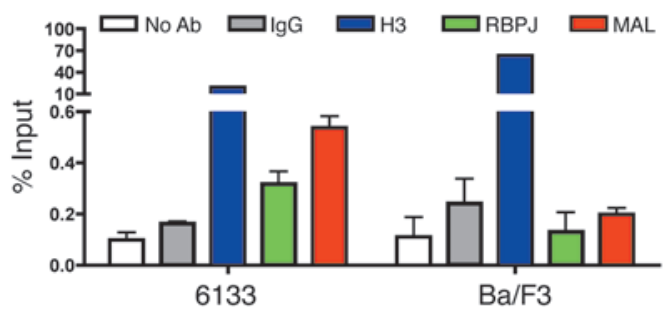

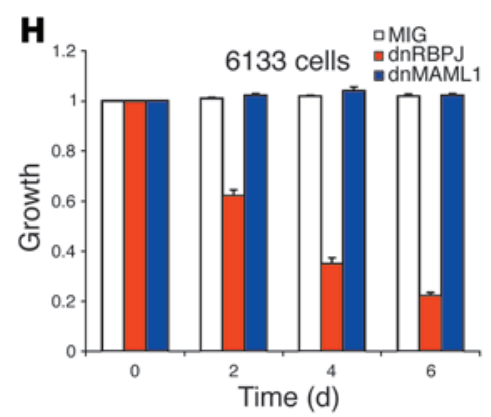

Figure 3

OTT-MAL activates RBPJ-mediated transcriptional activity. (A) Immunoprecipitation analyses in 293T cells transfected with OTT-MAL and OTT-MALARRM mutant show interaction between OTT-MAL and RBPJ through the N-terminal region of OTT (see B). STAT5 antibody was used as a nonspecific control. (B) Schematic representation of OTT, MAL, OTT-MAL (OM), and OM mutants. (C) 293T cells were transfected with the indicated constructs. Luciferase assays were performed 48 hours after transfection. Mean \pm SD light intensity of 3 independent experiments is shown. Inset, expression analysis of the different constructs in 293T cells by Western blot (anti-HA antibody). (D) TNR mice (GFP under the control of RBPJ-responsive elements) were crossed to OM animals, and GFP fluorescence intensity was analyzed in lineage-negative hematopoietic progenitors from WT, TNR, and OM+TNR mice. (E) RNA was extracted from lineage-negative cells of OM and WT littermate control animals and analyzed by quantitative RT-PCR for Notch target genes compared with Gapdh. Mean \pm SD of 3 independent experiments is shown. (F) Immunoprecipitation analyses of 6133 cell lysate indicate interaction between RBPJ and OTT-MAL in AMKL cells. (G) Chromatin immunoprecipitation assays were performed with 6133 cells and $\mathrm{Ba} / \mathrm{F} 3$ cells and analyzed by quantitative PCR using primers surrounding a RBPJ binding site in the endogenous promoter of the Hes1 gene. Mean \pm SD of triplicate quantitative PCR from a representative experiment are shown. (H) 6133 cells were transduced with a retrovirus expressing a dnRBPJ mutant. GFP-positive cells were flow sorted, and proliferation was monitored using trypan blue exclusion assay. Mean \pm SD relative to noninfected cells is represented $(n=3)$.

hematopoiesis in the spleen and liver $(6 / 15)$ and frank leukemia (2/15: nos. 6133 and 8904). These leukemias had phenotypic attributes of human AMKL, including infiltration of the BM, spleen, liver, and kidney with an admixture of immature megakaryocytic and erythroid elements as well as megakaryoblasts circulating in the peripheral blood (Supplemental Figure 2). The disease was transplantable into secondary and tertiary recipients, with a median survival of 45 days and 24 days, respectively (Figure 2D). Secondary recipients showed a phenotype similar to that of primary animals, with infiltrating leukemic blasts that were $\mathrm{vWF}^{+}$, confirming the megakaryoblastic nature of the disease (Figure 2E and Supplemental Figure 3).

A cytokine-dependent cell line was derived from leukemic cells of animal no. 6133 (Figure 2F). Similar to primary leukemic cells from this animal, the 6133 cell line displayed an immature phenotype (Figure $2 \mathrm{G}$ ). These cells were positive for c-Kit as well as several megakaryocytic markers, including CD41 and CD42b, and were negative for myeloid markers, including Mac1 and FceRIa 
A

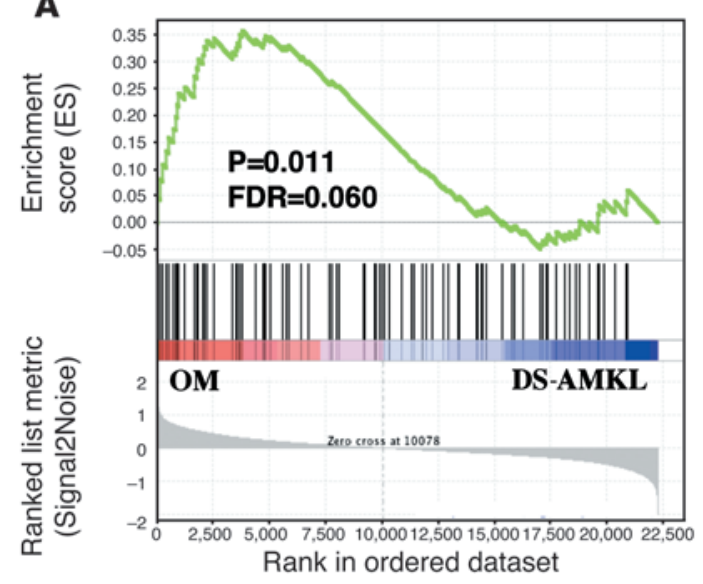

B

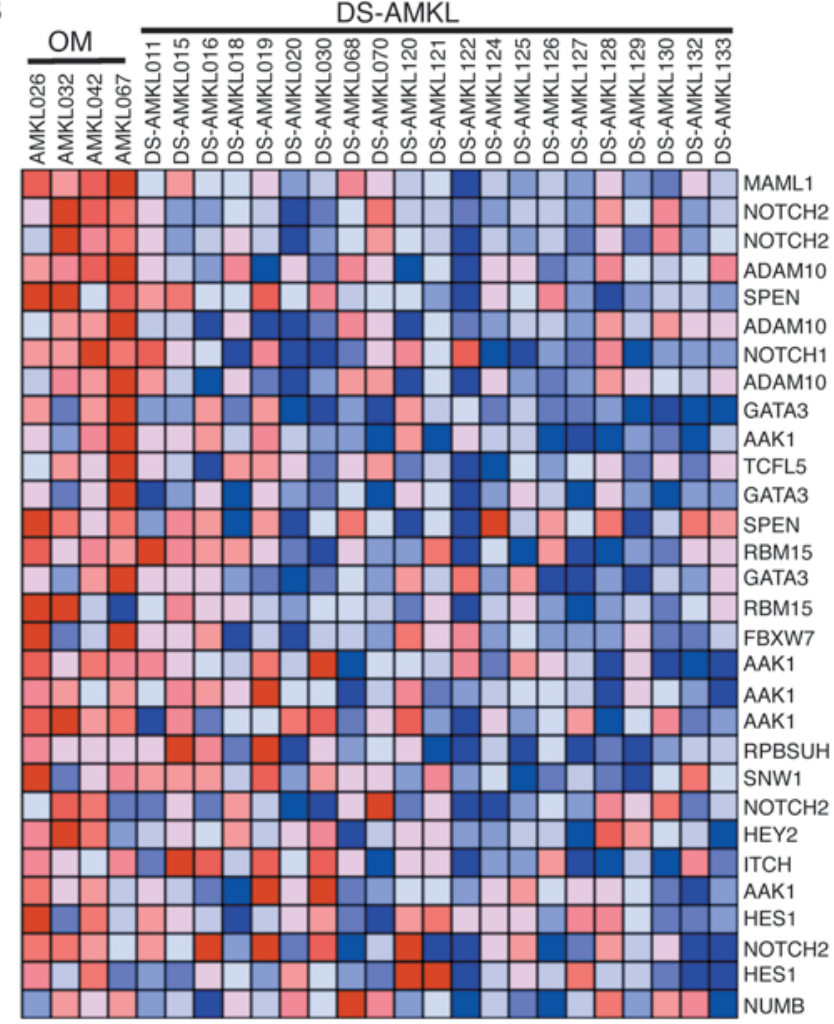

Figure 4

Gene expression analysis of human AMKL with $\mathrm{t}(1 ; 22)(\mathrm{p} 13 ; q 13)$ demonstrates a Notch pathway signature. (A) GSEA using a list of genes implicated in Notch signaling and comparing AMKL with $t(1 ; 22)(p 13 ; q 13)(O M-A M K L)$ with DS-AMKL. (B) Heat map representation of the 30 most enriched Notch genes in OM compared with DS-AMKL. Blue represents low expression; red represents high expression.

(Figure 2H). Together, these results indicate that expression of OTT-MAL results in abnormal differentiation of fetal hematopoietic progenitors, enhanced self-renewal properties of MkPs, and extramedullary hematopoiesis, leading to development of AMKL with low penetrance and long latency.

OTT-MAL activates RBPJ-mediated transcriptional activity. OTT belongs to a family of proteins that interact with the transcription factor RBPJ $(11-13,24)$, and constitutive activation of RBPJmediated transcription due to Notch signaling has been reported to result in increased self renewal and cancer $(25,26)$. To better understand the mechanism of megakaryocytic transformation by OTT-MAL, we determined whether OTT-MAL could interfere with RBPJ-mediated transcriptional regulation. Coimmunoprecipitation experiments in $293 \mathrm{~T}$ cells showed that OTT-MAL formed a complex with RBPJ that required the RNA recognition motif (RRM) domains of OTT-MAL (Figure 3, A and B). Furthermore, transactivation assays in $293 \mathrm{~T}$ cells using an RBPJ luciferase reporter showed that OTT-MAL activated RBPJ-mediated transcription in a dose-dependent manner, whereas expression of OTT resulted in a subtle inhibition of RBPJ transcription (Figure 3C) (13). Transactivation of the RBPJ reporter by OTT-MAL was dependent on the transactivation domain (TAD) of MAL and the RRM domains of OTT (Figure 3, B and C).

To investigate whether the RBPJ target genes could be transcriptionally activated by OTT-MAL in vivo, we first crossed OM animals with an established transgenic Notch reporter (TNR) mouse model, in which the expression of a GFP reporter is under the control of RBPJ response elements $(27,28)$. GFP fluorescence was higher in lineage-negative hematopoietic cells from double OM+TNR animals than in cells from control TNR animals (Figure 3D). In addition, primary lineage negative hematopoietic cells from OM animals showed increased expression of Hes1, Hes5, and Dtx1 transcripts compared with WT animals (Figure 3E), confirming activation of RBPJ transcription by OTT-MAL in vivo. Of note, Gata1 and Gata2 transcripts were also upregulated in OM versus WT cells (Supplemental Figure 4A). These results demonstrate increased RBPJ transcriptional activity in the hematopoietic progenitors of OM animals with respect to WT controls.

We next assessed whether OTT-MAL activation of RBPJ transcription is involved in the proliferation of 6133 AMKL cells. We performed coimmunoprecipitation to confirm that endogenous OTT-MAL but not MAL interacted with RBPJ in the $6133 \mathrm{AMKL}$ cell line (Figure 3F). In addition, chromatin immunoprecipitation analyses indicated that MAL and RBPJ were present at the HES1 promoter in 6133 cells but were not present at the HES1 promoter in control Ba/F3 cells that do not express OTT-MAL (Figure 3G). As confirmation, we also transduced 6133 cells with a dominant negative $(\mathrm{dn})$ form of RBPJ (dnRBPJ) that is unable to bind DNA (29) or with a dn Mastermind-like 1 (dnMAML1) mutant that entraps intracellular Notch (ICN) and RBPJ in transcriptionally inactive complexes when Notch signaling is activated $(30,31)$. Compared with empty vector control, dnRBPJ expression specifically inhibited the growth of 6133 AMKL cells but not control 
A

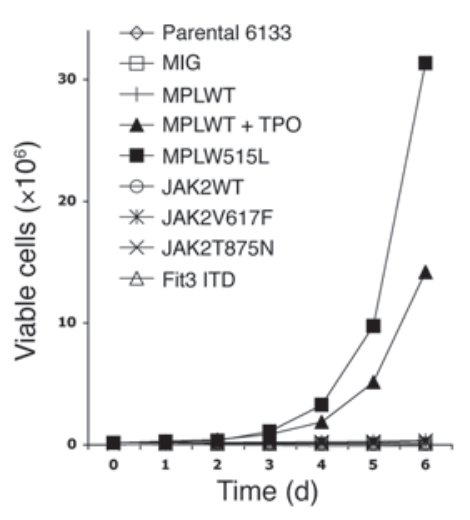

B

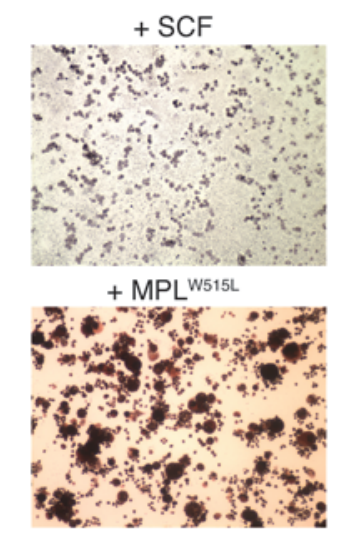

C
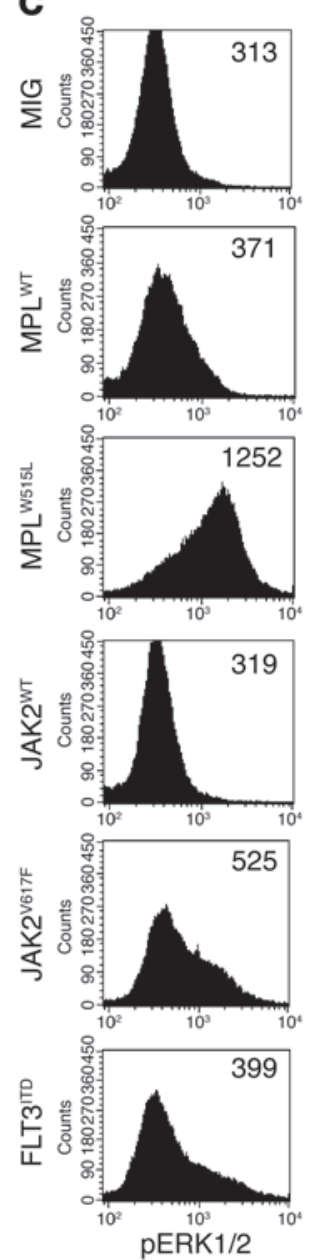

D

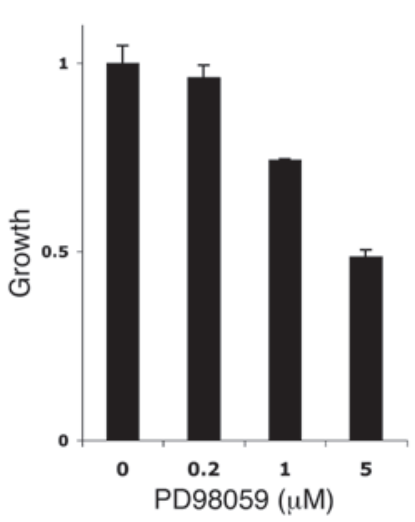

E

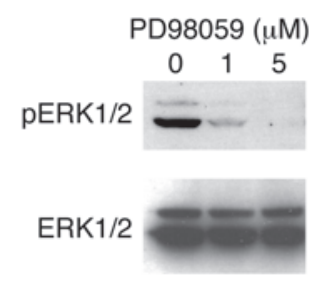

G

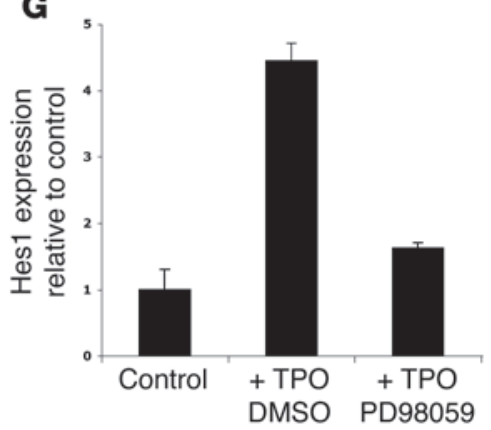

\section{Figure 5}

MPL mutant transforms 6133 AMKL cells. (A) Parental 6133 cells were transduced with empty vector (MIG) or JAK2 ${ }^{W T}, M P L^{W T}$, JAK2 ${ }^{V 617 F}$, $J A K 2^{T 875 N}, M P L^{W 515 L}$, and FLT3/TDN51 retroviruses. 24 hours after infection, cells were transferred to SCF-free medium, and viable cells were counted daily using a trypan blue exclusion assay. (B) 6133 cells growing with SCF stimulation or transduced with MPLW515L were cytospun on glass slides and stained for AchE. Representative pictures are shown. Original magnification, $\times 100$. (C) Flow cytometric analysis of phospho-ERK1/2 in 6133 cells, 48 hours after transduction with the different mutants and controls. Mean fluorescence intensity is indicated on the histograms. (D) Growth inhibition of $6133+\mathrm{Mp} / \mathrm{W515L}$ cells by MAPK inhibitor. The MAPK inhibitor PD98059 was used to treat $6133+\mathrm{MplW515L}$ cells. Growth at 48 hours compared with that of untreated cells is shown. Mean \pm SD of 3 independent experiments is shown. (E) ERK1/2 phosphorylation in $6133+\left.\mathrm{Mp}\right|^{\mathrm{W} 515 \mathrm{~L}}$ cells in presence of MAPK inhibitor. 6133+MplW515L cells were treated with PD98059, and cell lysates were used in direct Western blot for phospho-ERK1/2, followed by total ERK1/2. (F) 6133 cells transduced with WT MPL were maintained under SCF culture and then stimulated with TPO (10 ng/ml). RNA was extracted before TPO stimulation (control) and 2 hours after (+TPO). Expression of Hes1 and Gata1 transcripts was compared with Gapdh by quantitative RT-PCR. Mean \pm SD of 3 independent experiments is shown. (G) 6133 cells were analyzed as in $\mathbf{F}$ and treated with MAPK inhibitor (PD98059: $5 \mu \mathrm{M}$ ) during TPO stimulation. Mean \pm SD of 3 independent experiments is shown.

$\mathrm{Ba} / \mathrm{F} 3$ cells (Figure 3H and Supplemental Figure 4). In contrast, dnMAML1 or $\gamma$-secretase inhibitors had no significant effect on growth of 6133 or Ba/F3 cells (Supplemental Figure 4 and data not shown), suggesting that the expression of RBPJ target genes is not dependent on the activation of Notch receptors but is due to direct interaction between OTT-MAL and RBPJ.Taken together, these results indicate that RBPJ-mediated transcription is aberrantly induced by OTT-MAL in vitro and in vivo and is required for the growth of OTT-MAL-transformed AMKL cells.

An RBPJ pathway signature is upregulated in $t(1 ; 22)$ (p13;q13) $A M K L$. This mouse model expressing OTT-MAL indicates that aberrant activation of the RBPJ transcription factor, a media- tor of the canonical Notch signaling pathway, is important for leukemic transformation of the Mk lineage. To confirm the role of the pathway in human AMKL, we analyzed the expression of RBPJ pathway genes in human AMKL associated with $\mathrm{t}(1 ; 22)(\mathrm{p} 13 ; \mathrm{q} 13)$ (OM-AMKL) compared with DS-AMKL, the other molecularly defined subgroup of childhood AMKL. Using published global expression data from AMKL samples (32), we performed Gene Set Enrichment Analysis (GSEA) using a list of genes implicated in the RBPJ pathway (Supplemental Table 1). GSEA results showed that a RBPJ pathway signature was significantly enriched in OM-AMKL compared with DS-AMKL (Figure 4A). The most upregulated genes in OM-AMKL included Notch1, 
Notch2, MAML1, and RBPJ as well as direct RBPJ targets, including Hey2, Gata3, and TCFL5 (33-36) (Figure 4B). Together, these results show RBPJ pathway activation in human AMKL cells expressing the OTT-MAL fusion.

OTT-MAL cooperates with MPL signaling to induce AMKL in mice. Based on the low incidence of AMKL in animals expressing OTT-MAL alone, we hypothesized that deregulation of RBPJ transcription by OTT-MAL was not sufficient for development of AMKL and that cooperating oncogenic events were required. To identify such candidate events, we first used the 6133 cell line to screen cooperating mutations in cytokine receptors and signaling molecules that have been described in human Mk malignancies $(18,19,22,23)$. This included FLT3 $3^{\text {ITDN51 }}$ JAK2 $2^{V 617 F}$, JAK2 $2^{\text {T875N }}$, and $M P L^{W 515 L}$. We observed that $M P L^{W 515 L}$ transduction but not transduction with other alleles efficiently transformed 6133 cells, resulting in cytokine-independent growth (Figure 5A) and induced a megakaryocytic phenotype, as assessed in part by prominent staining for AchE (Figure 5B).

To compare the pathways activated by $M P L^{W 515 L}$ and by the other mutants in the context of OTT-MAL, we assessed phosphorylation levels of several relevant signaling transduction intermediates, including ERK1/2 (MAPK pathway), STAT3 and STAT5 (STAT pathway), and S6 (PI3K pathway) by flow cytometry on 6133 cells stably transduced with FLT3 $3^{I T D N 51}$, JAK2 $2^{V 617 F}, J A K 2^{T 875 N}$, or MPL W515L, respectively. ERK1/2 was markedly more activated by $M P L^{W 515 L}$ than by the other mutants, which did not confer factor-independent growth to 6133 cells (Figure 5C), whereas activation of the STAT or PI3K pathway was more uniform among all the mutants (Supplemental Figure 5). To further delineate the importance of the pathway activation in this context, we tested the effect of MAPK inhibitors. We observed that the MAPK inhibitor PD98059 inhibited the growth of 6133 cells expressing $M P L^{W 515 L}$ and ERK1/2 phosphorylation in a dose-dependent manner (Figure 5, D and E). Of note, stimulation of 6133 cells expressing the WT MPL receptor with TPO also induced proliferation (Figure 5A), indicating that proliferation was mediated by activation of the TPO/MPL signaling pathway rather than specifically by the MPL ${ }^{\mathrm{W} 515 \mathrm{~L}}$ mutant. Interestingly, TPO stimulation of 6133 cells expressing WT MPL led to a rapid increase in Hes 1 transcript but not Gata1 transcript (Figure 5F). This increase was inhibited by treatment with MAPK inhibitors (Figure 5G). Taken together, these results indicate that activation of the MPL/MAPK signaling pathway is important for megakaryoblastic transformation and increases HES1 transcription in the context of OTT-MAL.

To corroborate cooperativity between MPL signaling and OTT-MAL in primary hematopoietic cells in vivo, BM cells from 2-month-old nonleukemic OM or WT littermates were transduced with retroviruses harboring the MPL ${ }^{W 515 L}$ allele and transplanted into lethally irradiated WT C57BL/6 recipients (termed $\mathrm{OM}+M P L^{W 515 L}$ or $\mathrm{WT}+M P L^{W 515 L}$, respectively). In the $\mathrm{C} 57 \mathrm{BL} / 6$ background BM transplant model, $M P L^{W 515 L}$ induced an MPD with leukocytosis, polycythemia, and marked thrombocytosis similar to that reported in the BALB/c background (23), albeit with a longer latency (median $=60$ days; Supplemental Figure 6). In both the WT and OM contexts, the animals showed comparable degrees of leukocytosis, polycythemia, thrombocythemia, and splenomegaly, whereas the degree of hepatomegaly was significantly increased when OM cells were used (Figure 6, A and B, and Supplemental Figure 6). In contrast, when $M P L^{W 515 L}$ and OTT-MAL were coexpressed, flow cytometric analysis of spleno- cytes showed a significant shift toward immature progenitors as assessed by a marked increase in $\mathrm{c}-\mathrm{Kit}^{+}$staining in $\mathrm{OM}+M P L^{W 515 L}$ animals (Figure 6C and Supplemental Figure 7A), and the disease phenotype was more severe in this context, as indicated by a substantial increase in the number of CD41 ${ }^{+}, \mathrm{CD} 71^{+}$Ter119-, and $\mathrm{Mac1}^{+} \mathrm{Gr}^{+}$cells when compared with expression of $M P L^{W 515 L}$ alone. We next performed multiparameter flow analysis on BM or spleen cells, respectively, to phenotypically characterize hematopoietic progenitor populations. In contrast with WT+MPL W515L animals, which showed an increase in the common myeloid progenitor (CMP) population, $\mathrm{OM}+M P L^{W 515 L}$ animals showed a preferential increase in the Mk-erythrocyte progenitor (MEP) population in both BM and spleen (Figure 6D and Supplemental Figure 8). In addition, $\mathrm{MkP}$ that were specifically engaged in the Mk differentiation downstream of the MEP (37) were markedly expanded in $\mathrm{OM}+M P L^{W 515 L}$ animals compared with $\mathrm{WT}+M P L^{W 515 L}$ animals (Figure 6E and Supplemental Figure 8).

Histopathologic analysis of BM, spleen, and liver from WT $+M P L^{W 515 L}$ or $\mathrm{OM}+M P L^{W 515 L}$ animals showed infiltration with an admixture of megakaryocytic, erythroid, and myeloid elements (data not shown). Immunochemistry for vWF on spleen sections showed an increase in the number of mature polyploid Mks in $W T+M P L^{W 515 L}$ animals, whereas $\mathrm{OM}+M P L^{W 515 L}$ animals showed, in addition, the presence of numerous hypolobated immature megakaryoblasts (Figure 6F and Supplemental Figure 9). Together, these data demonstrate that in the WT C57BL/6 background, $M P L^{W 515 L}$ induces an MPD characterized by thrombocytosis and associated with expansion of the CMP population. In contrast, coexpression of MPL ${ }^{W 515 L}$ with OTT-MAL resulted in expansion of the MEP and $\mathrm{MkP}$ progenitor populations and evidence of transformation of the MPD phenotype into AMKL.

To further annotate malignant transformation, secondary transplantation was performed into sublethally irradiated recipients using $1 \times 10^{6}$ splenocytes from primary animals. Secondary $\mathrm{OM}+M P L^{W 515 L}$ animals developed a disease with leukocytosis and disease latency similar to that observed in the primary $\mathrm{OM}+M P L^{W 515 L}$ animals (Figure 7, A and C). However, in striking contrast with primary recipients, secondary recipients developed thrombocytopenia (Figure 7B). In control experiments, secondary $\mathrm{WT}+M P L^{W 515 L}$ or $\mathrm{OM}+M P L^{W T}$ animals showed no sign of disease for over 200 days, indicating that the $M P L^{W 515 L}$-induced MPD was not transplantable. Compared with control animals, flow cytometry analysis of secondary $\mathrm{OM}+M P L^{W 515 L}$ splenocytes showed abnormal CD $41^{+}, \mathrm{CD}_{42} \mathrm{~b}^{+}, \mathrm{CD} 41 / \mathrm{CD} 61^{+}$, or $\mathrm{CD}^{+}$populations, a fraction of which were also c-Kit ${ }^{\mathrm{lo} /+}$ (Figure 7D, Supplemental Figure $7 \mathrm{~B}$, and Supplemental Figure 10). These cells also expressed the CD71 marker but not Ter119, Mac1, or Gr1 markers. In agreement with these observations, histopathologic analysis demonstrated that BM, spleen, and liver were heavily infiltrated with immature hypolobated $\mathrm{vWF}^{+}$megakaryoblasts (Figure 7E and Supplemental Figure 10), with a striking reduction in median polyploidy (Figure 7F) and fibrosis (Figure 7G and Supplemental Figure 11). Southern blot analysis of DNA from BM cells showed over 10 viral integration sites in primary and secondary animals and suggests that the disease is oligo/polyclonal (Supplemental Figure 12). Transplantation into tertiary recipients resulted in a similar phenotype as in the secondary recipients of $\mathrm{OM}+M P L^{W 515 L}$ cells, albeit with a shorter latency (median = 38 days; Figure 7C). Collectively, these data show that coexpression of OTT-MAL and an activated MPL mutant induces $\mathrm{AMKL}$ with characteristic features of the human disease. 
A

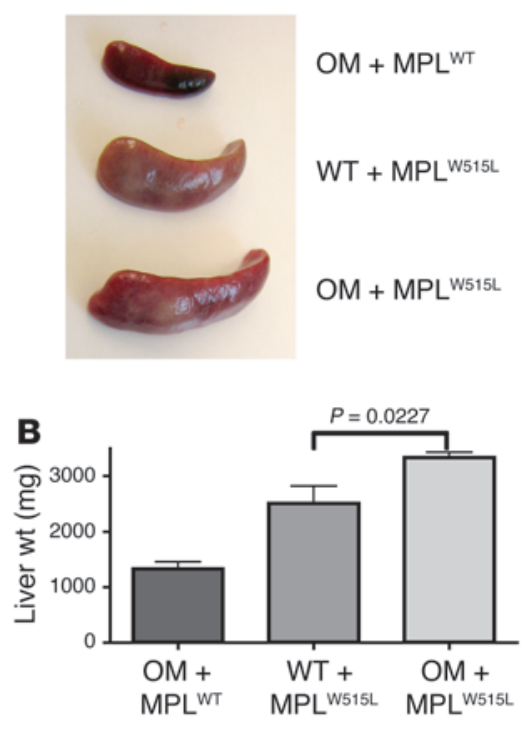

C

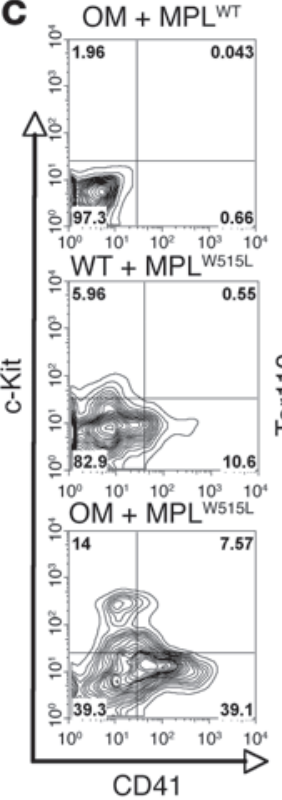

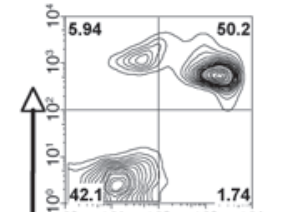
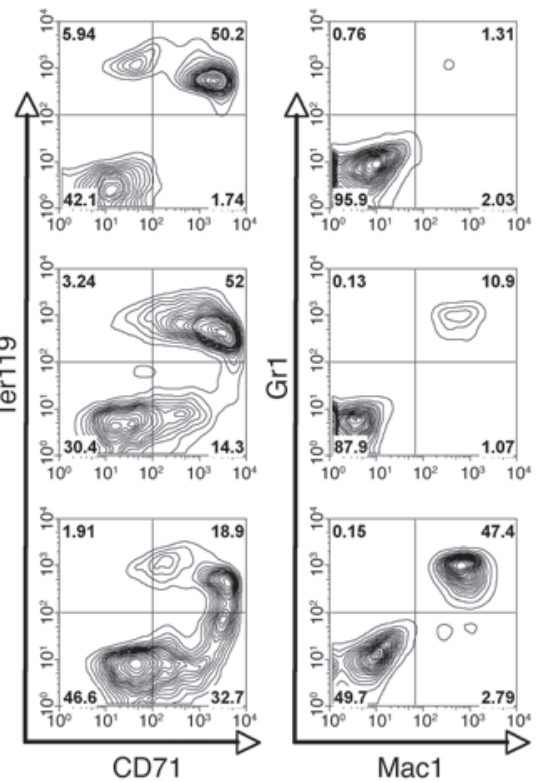

CD71

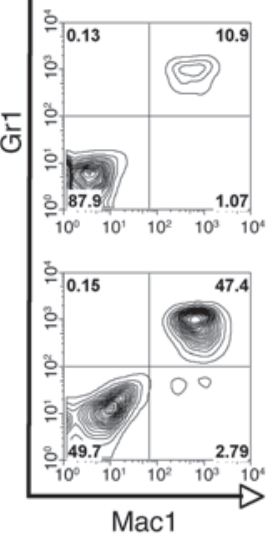

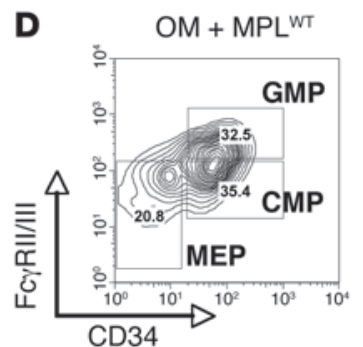
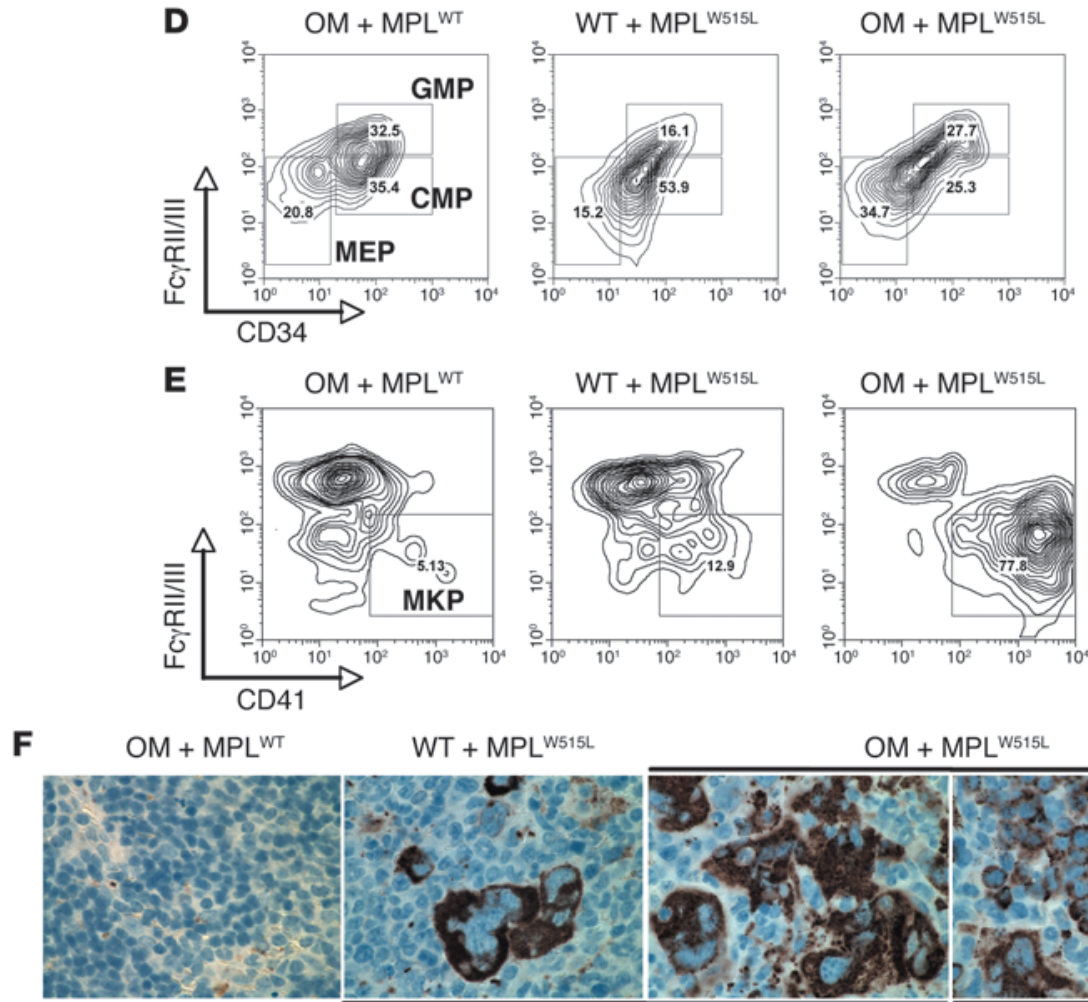

$\mathrm{OM}+\mathrm{MPL}{ }^{\mathrm{W}} 15 \mathrm{~L}$

Maturing Mks

Megakaryoblasts

Figure 6

Coexpression of MPLW515L with OTT-MAL results in AMKL in vivo. (A) Representative pictures of spleens from animals transplanted with OM or WT BM cells transduced with MPLW515L at 60 days after transplant. (B) Mean \pm SD of liver weights $(n=5)$. (C) Flow cytometry analysis of splenocytes at 60 days after transplantation. Experiments were performed in triplicate, and a representative analysis is shown. Percentages of live cells are indicated. (D) Multiparameter flow analysis of BM CMP, granulocyte-macrophage progenitor (GMP), and MEP populations. Experi-

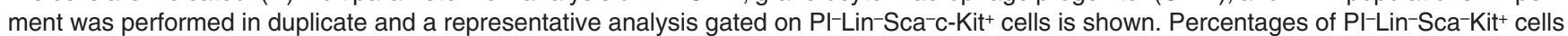
are indicated. (E) Multiparameter flow analysis of the BM MkP population. MkP are defined as $\mathrm{PI}-\mathrm{Lin}^{-} \mathrm{Kit}+\mathrm{CD}{ }^{+} \mathrm{FC} \gamma \mathrm{RII} / \mathrm{III}{ }^{\circ} \mathrm{CD} 41^{+}$. Percentages of Lin-Kit ${ }^{+} \mathrm{CD}{ }^{+}$cells are indicated. (F) vWF immunohistochemistry on spleen sections. Central panels show maturing polyploid Mks. Far right panel shows abnormal immature hypoploid Mks. Original magnification, $\times 1000$. 

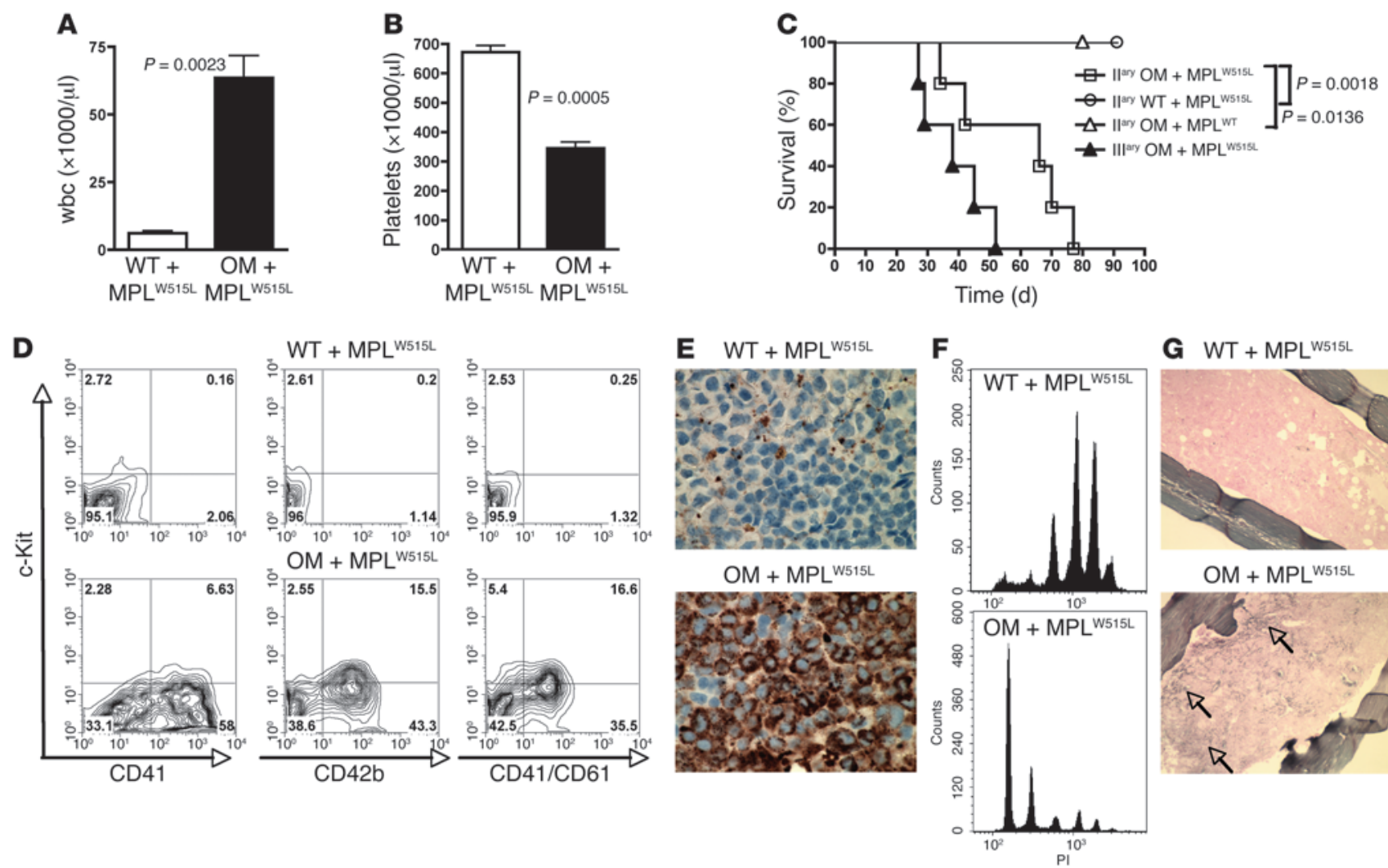

G $W T+M P L$ W515L
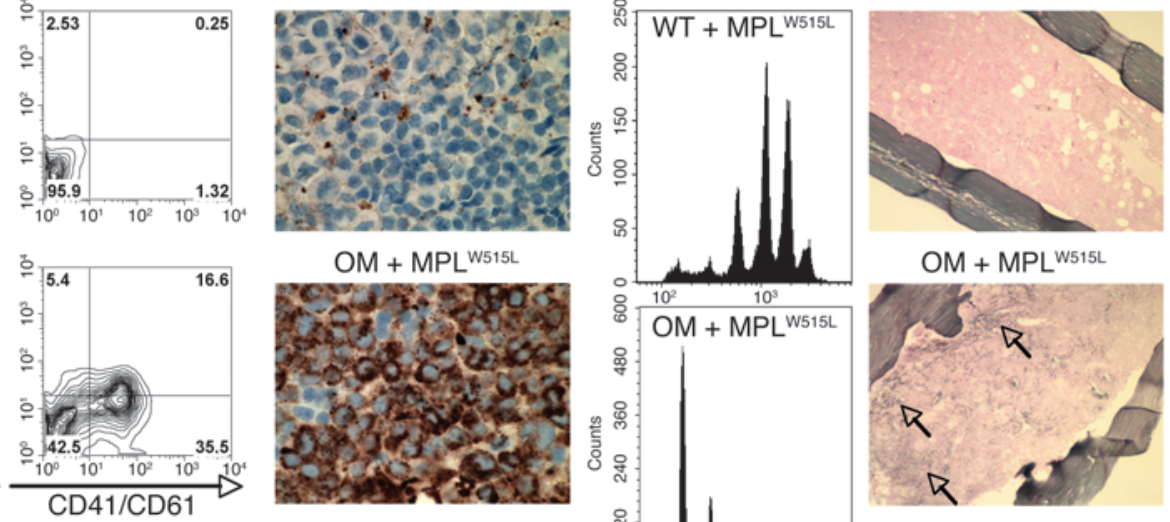

H

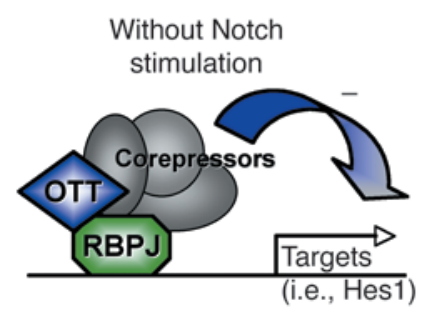

WT progenitors

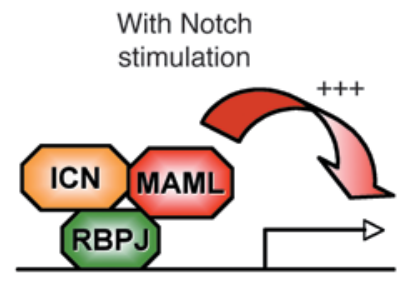

OTT-MAL progenitors

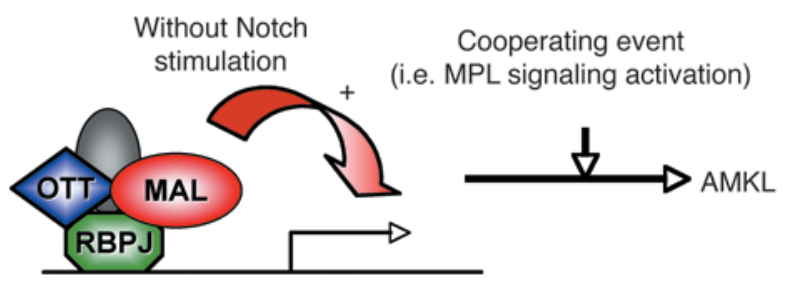

Figure 7

OTT-MAL+MPL W515L-induced AMKL shows characteristics of human AMKL. (A) White blood counts in secondary recipients. Mean \pm SD of 5 animals is shown. (B) Platelet counts in secondary recipients. Mean \pm SD of 5 animals is shown. (C) Kaplan-Meier survival curves of secondary and tertiary transplant recipients. (D) Flow cytometry analysis of splenocytes at 40 days after transplantation. Percentages of live cells are indicated. (E) VWF immunohistochemistry on spleen sections from secondary recipients. Original magnification, $\times 1000$. (F) Ploidy analysis was performed by flow cytometry using propidium iodide (PI). Histograms show a representative analysis gated on 10,000 CD41+ cells. (G) Histopathologic analysis of BM reticulin fibrosis in secondary recipients. Original magnification, $\times 100$. (H) Proposed model of leukemogenesis by OTT-MAL. In WT cells, repressor complexes are recruited to RBPJ in the absence of Notch stimulation. Following Notch stimulation, intracellular Notch (ICN) translocates to the nucleus, displaces corepressors, and binds RBPJ to allow recruitment of the coactivator MAML and expression of target genes. We propose that OTT-MAL aberrantly activates RBPJ-mediated transcription in the absence of Notch stimulation and leads to increased differentiation of hematopoietic progenitors to the Mk lineage. Full AMKL transformation requires additional cooperating events (i.e., activation of MPL signaling). Minus sign indicates inhibition of transcription; plus signs indicate activation of transcription.

\section{Discussion}

AMKL is generally associated with a poor prognosis, and its frequency may be underrepresented because of its complex clinical presentation and the difficulty in interpreting diagnostic material (38). Challenges in understanding the molecular basis of AMKL derive in part from the difficulty in obtaining adequate tissue samples derived from the fibrotic BM of patients. The identification of the OTT-MAL fusion in a subset of AMKL (5-7) provided a basis for development of a murine model of disease that could be used to increase understanding of the molecular pathogenesis of AMKL and potentially to provide insights into alternative therapeutic approaches.

We have observed that expression of OTT-MAL from its endogenous promoter results in abnormal megakaryopoiesis during embryonic and adult development but rarely causes AMKL. Several observations could explain the low efficiency of transformation of the Mk lineage in the context of the OTT-MAL fusion alone. It is possible that mutations associated with infant mega- 
karyoblastic leukemia preferentially target the fetal Mk compartment but have minimal phenotypic effects and transforming efficiency in the adult hematopoietic compartment. Biological differences between adult and fetal Mk precursors (39) as well as different microenvironmental influences may thus be crucial for transformation of the megakaryocytic lineage. In support of this hypothesis, other human Mk lineage disorders, including thrombocytopenia associated with the thrombocytopenia and aplasia of the radius (TAR) syndrome or the transient MPD in DS patients, are observed in infancy but often resolve spontaneously during childhood $(40,41)$. In addition, similar findings were reported for a Gata1s knockin mouse model, in which developmentally restricted abnormalities in megakaryopoiesis were observed but not leukemic transformation (42).

The observation that OTT-MAL animals develop malignancy at a low frequency underscores the requirement for cooperating mutations to achieve transformation to AMKL. In support of this hypothesis, several mutations in tyrosine kinases and signaling molecules have been identified in patients with AMKL and Mk malignancies $(18,19,22,23)$. We used a candidate gene approach to address the potential for cooperativity based on previously reported activating alleles in other megakaryocytic malignancies. Our data indicate that OTT-MAL cooperates only with a limited subset of activated signaling molecules. In particular, we observed that coexpression of OTT-MAL with an MPL mutant generates a fully penetrant and rapidly fatal disease closely resembling human AMKL. MPL is essential for normal Mk development, and activating MPL mutations are found in a spectrum of Mk disorders, including $\operatorname{AMKL}(21,23,43)$. Our results suggest that a basis for complementation of OTT-MAL with activated MPL might lie in the strong activation of the MAPK pathway. In keeping with this hypothesis, MAPK pathway activation has been shown to favor Mk differentiation (44, 45 ) and to control the activity of serum response factor and its cofactors $(8,9,46)$, suggesting that it could synergize with OTT-MAL. Together, these observations suggest that AMKL pathogenesis by OTT-MAL may be associated with pathogenetic events leading to the activation of the MAPK pathway.

One of the most intriguing pathogenetic insights gleaned from the OTT-MAL mouse model is the involvement of the RBPJ transcription factor in transformation of the megakaryocytic lineage through interaction with OTT-MAL and aberrant increases of RBPJ transcriptional activity. Activation of RBPJ-mediated transcription by OTT-MAL is also required for the growth of AMKL cells derived from leukemic animals. Thus, the transcriptional program engaged by RBPJ is important for sustaining the proliferation of OTT-MAL-transformed cells. Of note, OTT was recently reported to modulate Notch-induced transcriptional activation and thereby affect myeloid differentiation $(13,47)$. The $\mathrm{N}$-terminal portion of OTT, containing the RRM domains, is required for interaction of OTT-MAL with RBPJ, and the TAD of MAL (8) is necessary for activation of RBPJ transcription, suggesting that OTT-MAL aberrantly recruits transcriptional coactivators to regulatory elements of genes normally controlled by RBPJ in the absence of Notch signaling (Figure 7H). Although RBPJ activation by OTT-MAL may not strictly mimic activation by the canonical Notch pathway and the essential RBPJ targets for leukemogenesis by OTT-MAL remain to be identified, this mechanism is reminiscent of constitutive activation of RBPJ transcription by several viral proteins including EBNA2 (Epstein-Barr virus) and RTA (Kaposi virus) (48).
RBPJ is an essential mediator of the canonical Notch pathway that has been implicated in normal developmental processes (33, $49)$ and in cancer $(26,50)$. We have recently demonstrated that canonical Notch signaling specifies Mk fate of HSCs through RBPJ-dependent mechanisms (51). We now show that several RBPJ pathway genes are specifically upregulated in human AMKL patient cells associated with the $\mathrm{t}(1 ; 22)(\mathrm{p} 13 ; \mathrm{q} 13)$ as compared with DS-AMKL, the other subgroup of childhood AMKL. In our knockin model, we show that OTT-MAL-expressing hematopoietic progenitors have a differentiation bias toward the megakaryocytic lineage. Together, these results support the notion that aberrant activation of RBPJ transcription by OTT-MAL plays a role specifically in Mk transformation by skewing commitment of hematopoietic progenitors preferentially to the megakaryocytic lineage.

In toto, this knockin model of OTT-MAL expression provides important biological insights into what we believe is a novel mechanism of leukemogenesis of the Mk lineage and should be a useful tool for testing therapeutic approaches for the treatment of human AMKL that may include inhibition of RBPJ and the MAPK pathway, among others.

\section{Methods}

Targeting construct. A $15-\mathrm{kb}$ genomic DNA fragment containing the murine $\mathrm{Ott}$ locus was obtained by screening of a phage $\lambda$ mouse genomic DNA library. A 6.9-kb EcoRI-BamHI fragment representing the 5' homology arm was subcloned into a pBluescript KS vector along with a 5.4-kb BamHI-AvrII fragment representing the 3' homology arm. The human $M A L$ cDNA (NCBI nucleotide sequence number AJ297257) was obtained by screening of a phage $\lambda$ leukocytes cDNA library (Stratagene; gift from S. Gisselbrecht and G. Courtois, INSERM, Paris, France). An XhoI-BamHI fragment containing exons 4 to 15 of $M A L$ was introduced into the pBS302 vector (Life Technologies) in 3' of the transcriptional termination STOP cassette. A SalI-SalI fragment containing a loxP site was then introduced in between the STOP cassette and the MAL cDNA. A 2.1-kb EcoRI-HindIII fragment containing part of the Ott first intron with a BglII restriction enzyme site and $O t t$ second exon was then introduced in the pBS302 vector upstream of the STOP cassette. A BamHI-BamHI fragment containing a floxed Hygromycin resistance cassette was then introduced into the BglII site, creating a loxP-Hygromycin-loxP-Ott Exon2-loxP$M A L$ cDNA. A BamHI fragment containing this loxP-Hygromycin-loxPOtt Exon2-loxP-MAL cDNA was finally introduced into the BamHI site of the vector containing the $5^{\prime}$ and $3^{\prime}$ homology arms. All intermediates and final constructs were entirely resequenced.

ES cell culture and screening. A purified 21-kb Sall-NotI targeting fragment was electroporated into R1 ES cells by the Dartmouth College Transgenic Core (Dartmouth, New Hampshire, USA). For Southern blot analyses, genomic DNA from individual clones was digested with the restriction enzyme XmnI. Ott ${ }^{3 l o x / W T}$ ES cell clones were infected with an adenovirus encoding the Cre recombinase (MOI of 100). Fresh medium was added 2 hours later. 24 hours after infection, cells were trypsinized and plated at 1,000 cells $/ 100-\mathrm{mm}$ Petri dish. Individual colonies were replated after 5 days and expanded, and DNA was extracted for screening by Southern blot as described above. C57BL/6 blastocyst injection of ES cells presenting a normal karyotype was performed by the Brigham and Women's Hospital Transgenic Core. Chimeras were bred with C57BL/6 mice (Charles River) for germline transmission. Ott $3 l o x / W T$ animals were bred with EIIa-Cre transgenic mice (The Jackson Laboratory) to achieve transmission of the OTT ${ }^{O T T-M A L}$ allele through germline excision. Subsequently, OM mice were backcrossed at least 5 generations with WT C57BL/6 animals. 
Constructs. OTT, MAL, OTT-MAL, OTT-MALARRM, and OTTMAL $\triangle T A D$ were cloned into the PCMX-HA or pcDNA3-HA vector. All constructs were confirmed by resequencing. PJH23A (4xwtRBPJLuc) was a gift from S.D. Hayward (Johns Hopkins School of Medicine, Baltimore, Maryland, USA), and MPI79-HA-dnRBPJ (dnRBPJ) was a gift from O. Albagli (INSERM, Paris, France) and E. Lauret (INSERM, Paris, France) (29). The MSCV dnMAML1-GFP fusion was a gift from J.D. Griffin (Harvard Medical School, Boston, Massachusetts, USA) (31).

Notch reporter assay and TNR mice. Transfections were carried out using FuGENE (Roche) according to the manufacturer's protocols. For luciferase assays, HEK293 cells were plated in 6-well plates and transfected with $200 \mathrm{ng}$ pJH23A, $40 \mathrm{ng}$ pRL-TK, and 50 or $200 \mathrm{ng}$ of the indicated plasmids in a total of $500 \mathrm{ng}$ DNA. Luciferase activity was measured 48 hours later by Dual-Luciferase Reporter Assay Kit (Promega), and histograms show normalized results to pRL-TK luciferase. TNR mice $(27,28)$ expressing the eGFP under the control of 4 tandem copies of the RBPJ binding sites were obtained from The Jackson Laboratory (no. 005854) and crossed to $O M$ mice.

Microarray data analysis. For microarray analysis, we used a published data set of AMKLs (32) (Gene Expression Omnibus accession number GSE4119). We collected raw global gene expression values (from Affymetrix GeneChip U133A) for t 1 ;22)(p13;q13) AMKL (OM-AMKL) samples and DS-AMKL samples. We processed these samples with the robust multiarray analysis (RMA) algorithm (52) using BioConductor software, version 2.3 (53). Using the processed data, we then performed GSEA (http://www.broad.mit.edu/gsea/) (54) to look for enrichment of a list of Notch pathway genes (Supplemental Table 1). The GSEA algorithm identified a leading edge set of the genes that best distinguished OM-AMKL from DS-AMKL.

Immunoprecipitation and Western blot analysis. Cells were lysed in $20 \mathrm{mM}$ Tris, pH 7.4, 1\% Triton X-100, $150 \mathrm{mM} \mathrm{NaCl}, 1 \mathrm{mM}$ EDTA, 10\% glycerol (Complete; Roche), $1 \mathrm{mM}$ PMSF, $1 \mathrm{mM}$ sodium orthovanadate, and $0.1 \mathrm{mM}$ phenylarsine oxide. Lysates were normalized for protein content (BCA protein assay; Pierce, Thermo Scientific). For direct Western blot, $500 \mu \mathrm{g}$ (Figure 1) of total proteins was used following standard protocol. Anti-MAL antibodies (home-made no. 2346 directed against human MAL Ct portion or sc-21558; Santa Cruz Biotechnology Inc.) were used. For immunoprecipitation experiments, full-length or mutant HA-tagged OTT-MAL constructs were transfected into HEK293 cells. Protein lysates $(1,000 \mu \mathrm{g})$ collected 48 hours later were immunoprecipitated with either RBPJ (sc-28713; Santa Cruz Biotechnology Inc.) or STAT5 (sc-835; Santa Cruz Biotechnology Inc.). Eluate was analyzed by Western blot for HA (2362; Cell Signaling Technology) and RBPJ. $50 \mu \mathrm{g}$ of protein lysate was analyzed by direct Western blot to check for input. PD98059 (Calbiochem) was resuspended in DMSO, and an identical concentration of DMSO was used as control.

Chromatin immunoprecipitation analysis. $2 \times 10^{6} 6133$ or Ba/F3 cells were used for each chromatin immunoprecipitation, with $2 \mu \mathrm{g}$ of each antibody and following standard procedures.

Hes 1 promoter primers used for quantitative PCR were as follows: Hes1-F: GCAAAGCCCAGAGGAAAGAGTTAG; and Hes1-R: AGGAGAGAGGTAGACAGGGGATTC.

Flow cytometric analysis. Antibodies were purchased from BD except for FcyRII/III-PECy7 (Abcam) and goat anti-rabbit Alexa Fluor 647 (Molecular Probes; Invitrogen). Staining for flow cytometry was performed in $1 \times$ PBS with $2 \%$ FBS, except when otherwise mentioned. HSCs were stained for multiparameter flow analysis as previously described $(20,55)$, then analyzed on a highly modified double-laser (488-nm/350-nm, Enterprise II; and 647-nm, Spectrum; DakoCytomation) FACS (Moflo-MLS; DakoCytomation or FACSAria; BD Biosciences). Cell populations were defined as fol- lows: HSC, Lin-IL7R-Sca- ${ }^{+}{ }^{+} \mathrm{c}-\mathrm{Kit}^{+}$; CMP, $\mathrm{Lin}^{-} \mathrm{Sca}-1^{-} \mathrm{c}-\mathrm{Kit}^{+} \mathrm{CD} 34^{+} \mathrm{Fc} \gamma \mathrm{RII} / \mathrm{IIII}{ }^{\mathrm{lo}}$; granulocyte-macrophage progenitor (GMP), $\mathrm{Lin}^{-} \mathrm{Sca}-1^{-} \mathrm{c}-\mathrm{Kit}^{+} \mathrm{CD} 34^{+} \mathrm{Fc} \gamma \mathrm{RII} /$ III ${ }^{\text {hi; }}$ MEP, Lin-Sca-1-c-Kit ${ }^{+} \mathrm{CD} 34-\mathrm{Fc} \gamma \mathrm{RII} / \mathrm{III}^{\mathrm{lo}} .100 \mathrm{HSC}\left(\mathrm{Lin}^{-} \mathrm{Sca}-1^{+} \mathrm{c}-\mathrm{Kit}^{+}\right.$) were double sorted directly onto plates containing MethoCult M3434 (STEMCELL Technologies) supplemented with $10 \mathrm{ng} / \mathrm{ml} \mathrm{rhIL}-11,10 \mathrm{ng} / \mathrm{ml}$ rmGM-CSF, and $10 \mathrm{ng} / \mathrm{ml} \mathrm{rmTPO} \mathrm{(STEMCELL} \mathrm{Technologies).} \mathrm{Colonies}$ on triplicate plates were scored 7 days later.

$\mathrm{MkP}$ analysis was performed as previously described (37) with different fluorochrome-coupled antibodies to accommodate the presence of GFP in the cells: PECy5.5-conjugated lineage antibodies (Gr1, Ter119, CD19, B220, CD4, CD8, and CD3); biotin-CD9/APCCy7-avidin; CD41-PE; c-Kit-APC; and FcyRII/III-PECy7. MkPs were defined as Lin ${ }^{-}$ c- $\mathrm{Kit}^{+} \mathrm{CD}^{+} \mathrm{F} \gamma \gamma \mathrm{RII} / \mathrm{III}^{\mathrm{lo}} \mathrm{CD} 41^{+}$.

For ploidy analysis, cells were first stained with CD41 antibody, then stained with APC-conjugated secondary antibody. Cells were then incubated 30 minutes in a $0.1 \%$ sodium citrate solution containing $50 \mu \mathrm{g} / \mathrm{ml}$ of RNAse and $50 \mu \mathrm{g} / \mathrm{ml}$ of propidium iodide. Analysis of ploidy was performed using $1 \times 10^{4} \mathrm{CD} 41^{+}$cells. For phospho-flow analysis, 6133 cells were transduced with the different mutant viruses and analysis was performed 48 hours later. Cells were starved for 2 hours in RPMI 1640 plus 1\% BSA for 2 hours, fixed with $1 \%$ methanol-free formaldehyde (Polysciences Inc.), permeabilized with $100 \%$ methanol, and stained with phosphospecific antibodies (Cell Signaling Technologies): p-ERK1/2 (no. 9101), p-STAT3 (no. 9131), p-STAT5 (no. 9351), and p-S6 (no. 2211) followed by secondary goat anti-rabbit Alexa Fluor 647 antibody (Molecular Probes; Invitrogen). Analysis was gated on $\mathrm{GFP}^{+}$cells. Acquisition of the data was performed on a FACSCalibur (BD Biosciences); data were analyzed with FlowJo software, version 8.5.3.

Colony assays. Single-cell suspensions were prepared from E12.5 fetal livers by passing cells sequentially through 18 -gauge, 21-gauge, and 27-gauge needles. Cells were incubated with Red Blood Cells Lysis Buffer (Puregene; QIAGEN) for $5^{\prime}$ on ice and then resuspended in culture medium.

For myeloid colony assays, $2 \times 10^{4}$ cells were plated in MethoCult 3434 (STEMCELL Technologies) supplemented with TPO, and IL-11 and colonies were scored after 7 days. For Mk colony assays, $2.2 \times 10^{5}$ cells were mixed with MegaCult-C (STEMCELL Technologies) containing TPO, IL-11, IL-3, and IL-6, according to manufacturer's recommendation, and plated onto 2 double-chamber culture slides; colonies were enumerated after 8 days.

Staining and immunohistochemistry. AchE staining of MegaCult cultures or cytospun cells was performed following manufacturer's recommendations (STEMCELL Technologies). Blood smears were stained with Wright-Giemsa Stain (Sigma-Aldrich). H\&E staining, reticulin staining, and vWF (Dako) immunohistochemistry on paraffin-embedded tissue sections were performed by the Brigham and Women's Histopathology Core Facility following standard procedures. Images of histologic slides were obtained on a Nikon Eclipse E400 microscope equipped with SPOT RT color digital camera (model 2.1.1; Diagnostic Instruments Inc.). The microscope was equipped with a 10x/22 NA ocular lens. $\times 100$ images were obtained with a $10 x / 0.25$ objective lens, $\times 1000$ images were obtained with a 100x/1.4 objective lens with oil (Trak-300; Richard-Allan Scientific). Images were analyzed using Adobe Photoshop 6.0.

Transplantation and viral infection. $1 \times 10^{6}$ splenocytes from $6133\left(\right.$ OTT $\left.{ }^{O T T-M A L}\right)$ and 6127 (WT littermate) animals were injected into sublethally irradiated SCID recipients (C.B. 17 Fox Chase SCID, CD17SC.M; Taconic).

For cooperation studies, viral supernatants were obtained as described $(19,20) .6133$ cells were mixed with viral supernatants, spinfected for 90 minutes at $1800 \mathrm{~g}$, and returned to the incubator for 3 to 5 hours. 48 hours after infection, cells were transferred to SCF-free medium, and viable cells were counted every day using trypan blue staining. For BM transplant, 8- to 10-week-old OTT ${ }^{O T T-M A L}$ or WT littermate donor mice 
were injected with 5 -FU 5 days prior to BM collection. On day 0 , primary $\mathrm{BM}$ cells were obtained from femurs and tibiae and cultured overnight in RPMI 1640 supplemented with 10\% FBS+IL-3, IL-6, and SCF after lysis of red blood cells. Cells were mixed with identical titer viral supernatants 3 times on day 1 and day 2 and spinfected for 90 minutes at $1800 \mathrm{~g}$ each time. After the second spinfection, $1 \times 10^{6} \mathrm{cells}$ were injected in the tail veins of lethally irradiated $\mathrm{C} 57 \mathrm{BL} / 6$ recipients. Nonlethal eye bleeds were performed using EDTA or heparin-coated capillary tubes, and blood counts were performed within 30 minutes on a HemaVet HV950FS (Drew Scientific Group). Approval for the use of animals in this study was granted by the Children's Hospital Boston Animal Care and Use Committee (Boston, Massachusetts, USA).

Statistics. 2-tailed Student's $t$ tests were performed using the program GraphPad Prism, version 4.0c (GraphPad Software); $P<0.05$ was considered significant.

\section{Acknowledgments}

We are very grateful to Evelyne Lauret and Olivier Albagli for the generous gift of the dnRBPJ construct. We also thank Catherine Lavau, Richard Monni, Geneviève Courtois, Sebastien Malinge, Christine Ragu, Kelly Morgan, Guido Posern, Gerlinde Wernig, and Michael G. Kharas for helpful discussions; Martine Mauchauffe,
Michiko Kawakita, Sid Puram, and Rachel Okabe for technical support; and Jeffery L. Kutok and the Brigham and Women's Hospital Pathology Core Facility for histopathology analysis. This work was supported in part by NIH grants DK50654, CA66996, and U01 CA105423; The Leukemia and Lymphoma Society (to D.G. Gilliland); and INSERM and Ligue Nationale Contre le Cancer - Equipe Labellisée (to O.A. Bernard). D.G. Gilliland is a Doris Duke Foundation Distinguished Clinical Scientist and an investigator of the Howard Hughes Medical Institute. T. Mercher is the recipient of a Special Fellow Grant from The Leukemia and Lymphoma Society (3431-06).

Received for publication April 10, 2008, and accepted in revised form February 4, 2009.

Address correspondence to: Olivier A. Bernard, INSERM EMI0210, Tour Pasteur - Hôpital Necker, 149 rue de Sèvres, 75743 Paris Cedex 15, France. Phone: 33-1-44-49-58-63; Fax: 33-1-42-19-27-40; E-mail: olivier.bernard@inserm.fr. Or to: D. Gary Gilliland, Brigham and Women's Hospital, 1 Blackfan Circle, Boston, Massachusetts 02115, USA. Phone: (617) 355-9092; Fax: (617) 355-9093; E-mail: ggilliland@rics.bwh.harvard.edu.
1. Lion, T., et al. 1992. The translocation $\mathrm{t}(1 ; 22)(\mathrm{p} 13 ; \mathrm{q} 13)$ is a nonrandom marker specifically associated with acute megakaryocytic leukemia in young children. Blood. 79:3325-3330.

2. Dastugue, N., et al. 2002. Cytogenetic profile of childhood and adult megakaryoblastic leukemia (M7): a study of the Groupe Francais de Cytogenetique Hematologique (GFCH). Blood. 100:618-626.

3. Paredes-Aguilera, R., Romero-Guzman, L., LopezSantiago, N., and Trejo, R.A. 2003. Biology, clinical, and hematologic features of acute megakaryoblastic leukemia in children. Am. J. Hematol. 73:71-80.

4. Wechsler, J., et al. 2002. Acquired mutations in GATA1 in the megakaryoblastic leukemia of Down syndrome. Nat. Genet. 32:148-152.

5. Ma, Z., et al. 2001. Fusion of two novel genes, RBM15 and MKL1, in the $t(1 ; 22)(\mathrm{p} 13 ; \mathrm{q} 13)$ of acute megakaryoblastic leukemia. Nat. Genet. 28:220-221.

6. Mercher, T., et al. 2001. Involvement of a human gene related to the Drosophila spen gene in the recurrent $\mathrm{t}(1 ; 22)$ translocation of acute megakaryocytic leukemia. Proc. Natl. Acad. Sci. U. S. A. 98:5776-5779.

7. Mercher, T., et al. 2002. Recurrence of OTT-MAL fusion in $\mathrm{t}(1 ; 22)$ of infant AML-M7. Genes Chromosomes Cancer. 33:22-28.

8. Miralles, F., Posern, G., Zaromytidou, A.I., and Treisman, R. 2003. Actin dynamics control SRF activity by regulation of its coactivator MAL. Cell. 113:329-342.

9. Descot, A., et al. 2008. OTT-MAL is a deregulated activator of serum response factor-dependent gene expression. Mol. Cell. Biol. 28:6171-6181.

10. Ariyoshi, M., and Schwabe, J.W. 2003. A conserved structural motif reveals the essential transcriptional repression function of Spen proteins and their role in developmental signaling. Genes Dev. 17:1909-1920.

11. Oswald, F., et al. 2002. SHARP is a novel component of the Notch/RBP-Jkappa signalling pathway. EMBO J. 21:5417-5426.

12. Kuroda, K., et al. 2003. Regulation of marginal zone B cell development by MINT, a suppressor of Notch/RBP-J signaling pathway. Immunity. 18:301-312.

13. Ma, X., et al. 2007. Rbm15 modulates Notch-induced transcriptional activation and affects myeloid differentiation. Mol. Cell. Biol. 27:3056-3064.

14. Szalai, G., LaRue, A.C., and Watson, D.K. 2006. Molecular mechanisms of megakaryopoiesis. Cell. Mol. Life Sci. 63:2460-2476.

15. Deutsch, V.R., and Tomer, A. 2006. Megakaryocyte development and platelet production. Br.J. Haematol. 134:453-466.

16. Fishley, B., and Alexander, W.S. 2004. Thrombopoietin signalling in physiology and disease. Growth Factors. 22:151-155.

17. Kaushansky, K., and Drachman, J.G. 2002. The molecular and cellular biology of thrombopoietin: the primary regulator of platelet production. Oncogene. 21:3359-3367.

18. Jelinek, J., et al. 2005. JAK2 mutation 1849G>T is rare in acute leukemias but can be found in CMML, Philadelphia chromosome-negative CML, and megakaryocytic leukemia. Blood. 106:3370-3373.

19. Mercher, T., et al. 2006. JAK2T875N is a novel activating mutation that results in myeloproliferative disease with features of megakaryoblastic leukemia in a murine bone marrow transplantation model. Blood. 108:2770-2779.

20. Walters, D.K., et al. 2006. Activating alleles of JAK3 in acute megakaryoblastic leukemia. Cancer Cell. 10:65-75.

21. Malinge, S., et al. 2008. Activating mutations in human acute megakaryoblastic leukemia. Blood. 112:4220-4226.

22. James, C., et al. 2005. A unique clonal JAK2 mutation leading to constitutive signalling causes polycythaemia vera. Nature. 434:1144-1148.

23. Pikman, Y., et al. 2006. MPLW515L is a novel somatic activating mutation in myelofibrosis with myeloid metaplasia. PLoS Med. 3:e270.

24. Tsuji, M., Shinkura, R., Kuroda, K., Yabe, D., and Honjo, T. 2007. Msx2-interacting nuclear target protein (Mint) deficiency reveals negative regulation of early thymocyte differentiation by Notch/RBP-J signaling. Proc. Natl. Acad. Sci. U. S. A. 104:1610-1615.

25. Burns, C.E., Traver, D., Mayhall, E., Shepard, J.L., and Zon, L.I. 2005. Hematopoietic stem cell fate is established by the Notch-Runx pathway. Genes Dev. 19:2331-2342

26. Weng, A.P., et al. 2004. Activating mutations of
NOTCH1 in human T cell acute lymphoblastic leukemia. Science. 306:269-271.

27. Duncan, A.W., et al. 2005. Integration of Notch and Wntsignaling in hematopoieticstem cell maintenance. Nat. Immunol. 6:314-322.

28. Wu, M., et al. 2007. Imaging hematopoietic precursor division in real time. Cell Stem Cell. 1:541-554.

29. Lahmar, M., et al. 2007. Distinct effects of the soluble versus membrane-bound form of the notch ligand delta- 4 on human $\mathrm{Cd} 34+\mathrm{Cd} 38$ low cell expansion and differentiation. Stem Cells. 26:621-629.

30. Nam, Y., Sliz, P., Song, L., Aster, J.C., and Blacklow, S.C. 2006. Structural basis for cooperativity in recruitment of MAML coactivators to Notch transcription complexes. Cell. 124:973-983.

31. Weng, A.P., et al. 2003. Growth suppression of preT acute lymphoblastic leukemia cells by inhibition of notch signaling. Mol. Cell. Biol. 23:655-664.

32. Bourquin, J.P., et al. 2006. Identification of distinct molecular phenotypes in acute megakaryoblastic leukemia by gene expression profiling. Proc. Natl. Acad. Sci. U. S. A. 103:3339-3344.

33. Bray, S.J. 2006. Notch signalling: a simple pathway becomes complex. Nat. Rev. Mol. Cell Biol. 7:678-689.

34. Fang, T.C., et al. 2007. Notch directly regulates Gata3 expression during $\mathrm{T}$ helper 2 cell differentiation. Immunity. 27:100-110.

35. Amsen, D., et al. 2007. Direct regulation of Gata3 expression determines the $\mathrm{T}$ helper differentiation potential of Notch. Immunity. 27:89-99.

36. Weerkamp, F., et al. 2006. Identification of Notch target genes in uncommitted T-cell progenitors: No direct induction of a T-cell specific gene program. Lenkemia. 20:1967-1977.

37. Nakorn, T.N., Miyamoto, T., and Weissman, I.L. 2003. Characterization of mouse clonogenic megakaryocyte progenitors. Proc. Natl. Acad. Sci. U. S. A. 100:205-210.

38. Athale, U.H., et al. 2001. Biology and outcome of childhood acute megakaryoblastic leukemia: a single institution's experience. Blood. 97:3727-3732.

39. Debili, N., et al. 2001. Different expression of CD41 on human lymphoid and myeloid progenitors from adults and neonates. Blood. 97:2023-2030.

40. Letestu, R., et al. 2000. Existence of a differentiation blockage at the stage of a megakaryocyte pre- 
cursor in the thrombocytopenia and absent radii (TAR) syndrome. Blood. 95:1633-1641.

41. Zipursky, A. 2003. Transient leukaemia--a benign form of leukaemia in newborn infants with trisomy 21 . Br. J. Haematol. 120:930-938.

42. Li, Z., et al. 2005. Developmental stage-selective effect of somatically mutated leukemogenic transcription factor GATA1. Nat. Genet. 37:613-619.

43. Pardanani, A.D., et al. 2006. MPL515 mutations in myeloproliferative and other myeloid disorders: a study of 1182 patients. Blood. 108:3472-3476.

44. Rojnuckarin, P., Drachman, J.G., and Kaushansky, K. 1999. Thrombopoietin-induced activation of the mitogen-activated protein kinase (MAPK) pathway in normal megakaryocytes: role in endomitosis. Blood. 94:1273-1282.

45. Garcia, J., de Gunzburg, J., Eychene, A., Gisselbrecht, S., and Porteu, F. 2001. Thrombopoietin-mediated sustained activation of extracellular signal- regulated kinase in UT7-Mpl cells requires both Ras-Raf-1- and Rap1-B-Raf-dependent pathways. Mol. Cell. Biol. 21:2659-2670.

46. Muehlich, S., et al. 2008. Serum-induced phosphorylation of the serum response factor coactivator MKL1 by the extracellular signal-regulated kinase $1 / 2$ pathway inhibits its nuclear localization. $\mathrm{Mol}$. Cell. Biol. 28:6302-6313.

47. Raffel, G.D., et al. 2007. Ott1(Rbm15) has pleiotropic roles in hematopoietic development. Proc. Natl. Acad. Sci. U. S. A. 104:6001-6006.

48. Hayward, S.D. 2004. Viral interactions with the Notch pathway. Semin. Cancer Biol. 14:387-396.

49. Wilson, A., and Radtke, F. 2006. Multiple functions of Notch signaling in self-renewing organs and cancer. FEBS Lett. 580:2860-2868.

50. Roy, M., Pear, W.S., and Aster, J.C. 2007. The multifaceted role of Notch in cancer. Curr. Opin. Genet. Dev. 17:52-59.
51. Mercher, T., et al. 2008. Notch signaling specifies megakaryocyte development from hematopoietic stem cells. Cell Stem Cell. 3:314-326.

52. Irizarry, R.A., et al. 2003. Exploration, normalization, and summaries of high density oligonucleotide array probe level data. Biostatistics. 4:249-264.

53. Gentleman, R.C., et al. 2004. Bioconductor: open software development for computational biology and bioinformatics. Genome Biol. 5:R80.

54. Subramanian, A., et al. 2005. Gene set enrichment analysis: a knowledge-based approach for interpreting genome-wide expression profiles. Proc. Natl. Acad. Sci. U. S. A. 102:15545-15550.

55. Na Nakorn, T., Traver, D., Weissman, I.L., and Akashi, K. 2002. Myeloerythroid-restricted progenitors are sufficient to confer radioprotection and provide the majority of day 8 CFU-S. J. Clin. Invest. 109:1579-1585. 